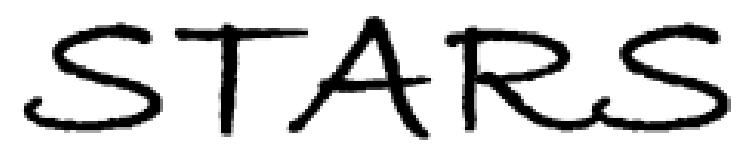

University of Central Florida

STARS

$1-1-2007$

\title{
Exact controllability for multidimensional semilinear hyperbolic equations
}

Xiaoyu Fu

Jiongmin Yong

University of Central Florida

Xu Zhang

Find similar works at: https://stars.library.ucf.edu/facultybib2000

University of Central Florida Libraries http://library.ucf.edu

This Article is brought to you for free and open access by the Faculty Bibliography at STARS. It has been accepted for inclusion in Faculty Bibliography 2000 s by an authorized administrator of STARS. For more information, please contact STARS@ucf.edu.

\section{Recommended Citation}

Fu, Xiaoyu; Yong, Jiongmin; and Zhang, Xu, "Exact controllability for multidimensional semilinear hyperbolic equations" (2007). Faculty Bibliography 2000s. 7131.

https://stars.library.ucf.edu/facultybib2000/7131

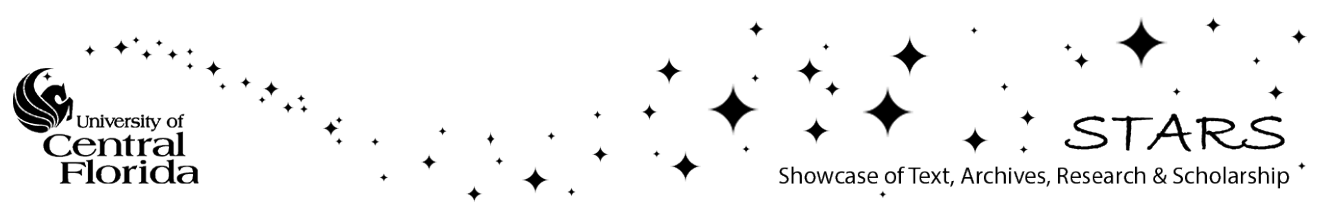




\title{
EXACT CONTROLLABILITY FOR MULTIDIMENSIONAL SEMILINEAR HYPERBOLIC EQUATIONS*
}

\author{
XIAOYU FU ${ }^{\dagger}$, JIONGMIN YONG ${ }^{\ddagger}$, AND XU ZHANG§
}

\begin{abstract}
In this paper, we obtain a global exact controllability result for a class of multidimensional semilinear hyperbolic equations with a superlinear nonlinearity and variable coefficients. For this purpose, we establish an observability estimate for the linear hyperbolic equation with an unbounded potential, in which the crucial observability constant is estimated explicitly by a function of the norm of the potential. Such an estimate is obtained by a combination of a pointwise estimate and a global Carleman estimate for the hyperbolic differential operators and analysis on the regularity of the optimal solution to an auxiliary optimal control problem.
\end{abstract}

Key words. exact controllability, semilinear hyperbolic equation, superlinear growth, observability inequality, global Carleman estimate

AMS subject classifications. Primary, 93B05; Secondary, 93B07, 35B37

DOI. $10.1137 / 040610222$

1. Introduction. Given $T>0$ and a bounded domain $\Omega$ of $\mathbb{R}^{n}(n \in \mathbb{N})$ with $C^{2}$ boundary $\Gamma$, put $Q=(0, T) \times \Omega$ and $\Sigma=(0, T) \times \Gamma$. Let $\omega$ be a proper open nonempty subset of $\Omega$ and denote by $\chi_{\omega}$ the characteristic function of $\omega$. For any set $M \subset \mathbb{R}^{n}$ and $\delta>0$, we define $\mathcal{O}_{\delta}(M)=\left\{x \in \mathbb{R}^{n}|| x-x^{\prime} \mid<\delta\right.$ for some $\left.x^{\prime} \in M\right\}$. Also, we denote $\sum_{i, j=1}^{n}$ and $\sum_{i=1}^{n}$ simply by $\sum_{i, j}$ and $\sum_{i}$, respectively. For simplicity, we will use the notation $y_{i}=y_{x_{i}}$, where $x_{i}$ is the $i$ th coordinate of a generic point $x=\left(x_{1}, \ldots, x_{n}\right)$ in $\mathbb{R}^{n}$. In a similar manner, we use the notation $w_{i}, v_{i}$, etc. for the partial derivatives of $w$ and $v$ with respect to $x_{i}$. On the other hand, for any domain $M$ in $\mathbb{R}^{n}$ (even without any regularity condition on its boundary $\partial M$ ), we refer to [1, Chap. 3$]$ for the definition and basic properties of the Sobolev spaces $H_{0}^{1}(M), H^{-1}(M)$, etc. (Hence, $H_{0}^{1}(Q)$ and $H^{-1}(Q)$ are particularly well defined in [1, Chap. 3].)

Let $a^{i j}(\cdot) \in C^{1}(\bar{\Omega})$ be fixed, satisfying

$$
a^{i j}(x)=a^{j i}(x) \quad \forall x \in \bar{\Omega}, \quad i, j=1,2, \ldots, n,
$$

and for some constant $\beta>0$,

$$
\sum_{i, j} a^{i j}(x) \xi^{i} \xi^{j} \geq \beta|\xi|^{2} \quad \forall(x, \xi) \in \bar{\Omega} \times \mathbb{R}^{n},
$$

where $\xi=\left(\xi^{1}, \ldots, \xi^{n}\right)$. In what follows, put $A \triangleq\left(a^{i j}\right)_{n \times n}$. We define a differential

${ }^{*}$ Received by the editors June 19, 2004; accepted for publication (in revised form) March 7, 2007; published electronically October 17, 2007. This work was partially supported by NSFC grants 10131030 and 10525105, Chinese Education Ministry Science Foundation grant 2000024605, the Cheung Kong Scholars Programme, NSF grant DMS-0604309, NCET of China grant NCET-040882, and Spanish MEC grant MTM2005-00714.

http://www.siam.org/journals/sicon/46-5/61022.html

†School of Mathematics, Sichuan University, Chengdu 610064, China (rj_xy@163.com).

${ }^{\ddagger}$ Department of Mathematics, University of Central Florida, Orlando, FL 32816, and School of Mathematical Sciences, Fudan University, Shanghai 200433, China (jyong@mail.ucf.edu).

$\S$ Yangtze Center of Mathematics, Sichuan University, Chengdu 610064, China, and Key Laboratory of Systems and Control, Academy of Mathematics and Systems Sciences, Chinese Academy of Sciences, Beijing 100080, China (xuzhang@amss.ac.cn).

1578 
operator $\mathcal{P}$ by

$$
\mathcal{P} y \triangleq y_{t t}-\sum_{i, j}\left(a^{i j}(x) y_{i}\right)_{j}
$$

Next, we fix a function $f(\cdot) \in C^{1}(\mathbb{R})$, satisfying the following condition:

$$
\varlimsup_{s \rightarrow \infty} \frac{f(s)}{s \ln ^{1 / 2}|s|}=0 .
$$

Note that $f(\cdot)$ in the above can have a superlinear growth. We consider the following controlled semilinear hyperbolic equation with an internal local controller acting on $\omega:$

$$
\begin{cases}\mathcal{P} y=f(y)+\chi_{\omega}(x) \gamma(t, x) & \text { in } Q \\ y=0 & \text { on } \Sigma \\ y(0)=y_{0}, \quad y_{t}(0)=y_{1} & \text { in } \Omega .\end{cases}
$$

In (1.5), $\left(y(t, \cdot), y_{t}(t, \cdot)\right)$ is the state, and $\gamma(t, \cdot)$ is the control which acts on the system through the subset $\omega$ of $\Omega$. In what follows, we choose the state space and the control space of system $(1.5)$ to be $H_{0}^{1}(\Omega) \times L^{2}(\Omega)$ and $L^{2}((0, T) \times \omega)$, respectively. We point out that some other choices of spaces are possible. But our choice is natural in the context of the hyperbolic equations. The space $H_{0}^{1}(\Omega) \times L^{2}(\Omega)$ is often referred to as the finite energy space. For any $\left(y_{0}, y_{1}\right) \in H_{0}^{1}(\Omega) \times L^{2}(\Omega)$ and $\gamma \in L^{2}((0, T) \times \Omega)$, using the method in [4] one can prove the global existence of a unique weak solution $y \in C\left([0, T] ; H_{0}^{1}(\Omega)\right) \cap C^{1}\left([0, T] ; L^{2}(\Omega)\right)$ for (1.5) under assumption (1.1)-(1.2), and under $(1.4)$ with $f(\cdot) \in C^{1}(\mathbb{R})$.

The main purpose of this paper is to study the global exact controllability of (1.5), by which we mean the following: For any given $\left(y_{0}, y_{1}\right),\left(z_{0}, z_{1}\right) \in H_{0}^{1}(\Omega) \times L^{2}(\Omega)$, find a control $\gamma \in L^{2}((0, T) \times \omega)$ such that the corresponding weak solution $y$ of $(1.5)$ satisfies

$$
y(T)=z_{0}, \quad y_{t}(T)=z_{1} \quad \text { in } \Omega .
$$

Due to the finite propagation speed of solutions to hyperbolic equations, the "waiting time" $T$ has to be large enough. The estimate of $T$ is also a part of the problem.

The problem of exact controllability for linear hyperbolic equations (for example, $f(\cdot)$ is a linear function, or simply, $f(\cdot) \equiv 0$ in $(1.5))$ has been studied by many authors. We mention here some standard references, for example, [2, 29, 33].

The study of exact controllability problems for nonlinear hyperbolic equations began in the 1960s. Early works, including $[5,6,10]$ and so on, were mainly devoted to the local controllability problem, by which we mean that the controllability property was proved under some smallness assumptions on the initial data and/or the final target. In [43], further local results were proved for the exact controllability of some semilinear wave equations in the form of (1.5) with $A=I$, the identity matrix, and under a very general assumption on the nonlinearity $f(\cdot)$ (which allows $f(\cdot)$ to be local Lipschitz continuous). We refer to [27] and the references cited therein for some recent local controllability results of certain quasi-linear hyperbolic systems.

A global boundary exact controllability result for semilinear wave equations, corresponding to (1.5), in the state spaces $H_{0}^{r}(\Omega) \times H^{r-1}(\Omega)(r \in(0,1 / 2) \cup(1 / 2,1))$ or $H_{00}^{1 / 2}(\Omega) \times\left(H_{00}^{1 / 2}(\Omega)\right)^{\prime}$, with Dirichlet boundary control, was given in [44] under the 
assumption that $A=I$ and that the nonlinearity $f(\cdot)$ is globally Lipschitz continuous, i.e., $f^{\prime}(\cdot) \in L^{\infty}(\mathbb{R})$. In [23], this controllability result was improved to include the critical points $r=0$ and 1 , and also extended to the abstract setting. Recent progress in this respect can be found in [36] and [37]. In the case that $f(\cdot)$ is sublinear, we refer to [34] for the global exact controllability of (1.5).

As for the case that $f(\cdot)$ grows superlinearly at infinity, very little is known for the global exact controllability of the semilinear hyperbolic equation (1.5) except for the one space dimension, i.e., $n=1$. We refer to $[3,9,30,45]$ for related one-dimensional results. To our best knowledge, in the superlinear setting, [26] is the only paper that discussed the global exact controllability for multidimensional system (1.5) (we refer to [42] for an updated survey on this problem). By assuming that $A=I$ and $\omega=\mathcal{O}_{\delta}(\Gamma) \cap \Omega$ for some $\delta>0,[26]$ shows that system (1.5) with $f(\cdot)$ satisfying (1.4) is exactly controllable. In this paper, based on a method which is different from [26], we shall consider a more general case by using a smaller controller $\omega=\mathcal{O}_{\delta}\left(\Gamma_{+}\right) \cap \Omega$ (see (2.5) for $\Gamma_{+}$) and allowing the coefficients matrix $A$ to be nonconstant one. We refer the reader to Condition 2.1 and the subsequent remarks, and especially Proposition 2.1, for assumptions on matrix $A$.

In order to obtain the exact controllability of (1.5), one needs to consider, by the well-known duality argument (see [29], [28, Lemma 2.4, p. 282], and [39, Theorem 3.2, p. 19], for example), the following dual system of the linearized system of (1.5):

$$
\begin{cases}\mathcal{P} w=q w & \text { in } Q \\ w=0 & \text { on } \Sigma \\ \left(w(0), w_{t}(0)\right)= & \left(w_{0}, w_{1}\right) \in L^{2}(\Omega) \times H^{-1}(\Omega)\end{cases}
$$

with a potential $q$ in some space (larger than $L^{\infty}(Q)$, in general). It follows from the standard perturbation theorem in the semigroup theory [31] that for a suitable $q$, say $q \in L^{\infty}\left(0, T ; L^{n}(\Omega)\right),(1.7)$ is well-posed in $L^{2}(\Omega) \times H^{-1}(\Omega)$.

Similar to [45] and [26], the above controllability problem may be reduced to an explicit observability estimate for system (1.7). Namely, we expect to find a constant $\mathcal{C}(q)>0$ such that all weak solutions $w$ of (1.7) satisfy

$$
\left|\left(w_{0}, w_{1}\right)\right|_{L^{2}(\Omega) \times H^{-1}(\Omega)} \leq \mathcal{C}(q)|w|_{L^{2}((0, T) \times \omega)} \forall\left(w_{0}, w_{1}\right) \in L^{2}(\Omega) \times H^{-1}(\Omega) .
$$

The explicit estimate of $\mathcal{C}(q)$ in terms of a suitable norm of the potential $q$ is an indispensable part of the problem, which is actually the key novelty in this paper. Similar problems for $A=I$ and bounded potentials $q$ were considered in [36, 37]. However, in the present case we cannot assume that $q$ in (1.7) is bounded since we do not assume that the nonlinearity $f(\cdot)$ in $(1.5)$ is globally Lipschitz continuous. To overcome this difficulty, we need, among other things, to combine some ideas found in [18] and [37].

It is well known that the Carleman estimate is one of the major tools used in the study of unique continuation, observability, and controllability problems for various kinds of partial differential equations (PDEs). However, the "concrete" Carleman estimate for these problems is actually quite different! Indeed, in principle, among these problems unique continuation is the "easiest," and one may develop an abstract theory for the unique continuation property (usually, of local nature) for very general partial differential operators, based on a pseudoconvexity condition, the Carleman estimate, and by means of the microlocal analysis technique [16, 17, 35]. Observability is, however, a quantitative version of the global unique continuation, which is much 
more difficult to establish than the classical (qualitative) unique continuation. For example, the unique continuation for the parabolic equations was known for a very long time, but the observability for the same equation was not established until the 1990s by means of a new Carleman estimate [11, 14]. Also, for the hyperbolic equations, the work of $[20,21]$ applied Carleman estimates for the proofs of the observability results. On the other hand, there are many equations (say, the hyperbolic-parabolic coupled systems in [32]), for which one can easily establish its unique continuation, but its observability is completely unknown for multidimensions (the analysis for the one-dimensional problem [41] is highly nontrivial, and some atypical phenomenon occurs). Finally, as for controllability problems, as mentioned before the classical duality argument reduces the problem to obtaining a suitable observability estimate. However, for the global controllability problems for semilinear PDEs with superlinear growing nonlinearity, the key point is the explicit estimate of the observability constant by a suitable function of the norm of the potential. For this purpose, one has to proceed more carefully than one would for the usual observability when using the Carleman estimate. Note also that the approach developed in this article seems to be virtually complete. Our key estimate on the observability constant $\mathcal{C}(q)$ is presented in (2.12) of Theorem 2.3. As suggested by [8, Theorem 1.2], it may well be that (2.12) is sharp (see also our Remark 2.1). In this respect, it is worth mentioning that one can also adopt the method developed in $[20,21,22]$ to establish an explicit observability estimate for some special case of system (1.7) (i.e., $A=a I$ with a suitable positive function $a$ ), as done in [36]. However, it seems that the estimate obtained in this way is far from sharp. Indeed, the estimate on the observability constant $\mathcal{C}(q)$ obtained in [36] (for bounded potential $q$ ) reads as $C \exp \left(\exp \left(\exp \left(C r_{0}\right)\right)\right.$ ) with $r_{0}=|q|_{L^{\infty}(Q)}$, which is much weaker than that in (2.12). It would be quite interesting to check whether the method in $[20,21,22]$ can be adopted to derive the same estimate as that of (2.12) in Theorem 2.3. But this remains to be done.

The rest of this paper is organized as follows. In section 2 , we shall state the main results. Some preliminary results are collected in section 3 . In section 4 , we derive an estimate for second order differential operators with symmetric coefficients that is of independent interest. This estimate will play a key role when we establish in section 5 a global Carleman estimate for the hyperbolic differential operators in $H_{0}^{1}(Q)$. The latter estimate, in turn, is one of the crucial preliminary results we derive in section 7, i.e., a similar global Carleman estimate for the hyperbolic differential operators in a larger space $L^{2}(Q)$. Another crucial preliminary we study, in section 6 , is an auxiliary optimal control problem, where the key point is to obtain some regularity of the optimal solution. In sections $8-9$, we will prove our main results. Finally, Appendices A, B, and C are devoted to proving some technical results that are used throughout the paper.

2. Statement of the main results. To begin, we introduce the following condition.

Condition 2.1. There exists a function $d(\cdot) \in C^{2}(\bar{\Omega})$ satisfying the following: (i) For some constant $\mu_{0}>0$, it holds that

$$
\begin{array}{r}
\sum_{i, j}\left\{\sum_{i^{\prime}, j^{\prime}}\left[2 a^{i j^{\prime}}\left(a^{i^{\prime} j} d_{i^{\prime}}\right)_{j^{\prime}}-a_{j^{\prime}}^{i j} a^{i^{\prime} j^{\prime}} d_{i^{\prime}}\right]\right\} \xi^{i} \xi^{j} \geq \mu_{0} \sum_{i, j} a^{i j} \xi^{i} \xi^{j} \\
\forall\left(x, \xi^{1}, \ldots, \xi^{n}\right) \in \bar{\Omega} \times \mathbb{R}^{n} .
\end{array}
$$

Copyright (c) by SIAM. Unauthorized reproduction of this article is prohibited. 
(ii) There is no critical point of function $d(\cdot)$ in $\bar{\Omega}$, i.e.,

$$
\min _{x \in \bar{\Omega}}|\nabla d(x)|>0 .
$$

Let us make some remarks on the above condition.

First, Condition 2.1 is really a restriction on the coefficient matrix $A$ and the domain $\Omega$. Indeed, as we shall see later, Condition 2.1 at least leads to the exact controllability of system (1.5) with $f(\cdot) \equiv 0$ and $\omega=\mathcal{O}_{\delta}(\Gamma) \bigcap \Omega$ for any given $\delta>0$ and sufficiently large "waiting time" $T>0$, while it is shown in [2] that, in order for the latter to hold, $(T, \Omega, \omega)$ has to satisfy a geometric optics condition which is characterized by the null bicharacteristic of operator $\mathcal{P}$. But, for any $T>0$, this condition may fail to be true for some $\mathcal{P}$ (with special coefficients) and some $(\Omega, \omega)$ (see [2]). This condition is crucial in what follows, where we derive a Carleman estimate for the hyperbolic operators (see (11.4)). Nevertheless, to the best of our knowledge, there is no universal tractable Carleman estimates in the literature for general hyperbolic operators. We shall give below some tractable examples. However, a detailed analysis of Condition 2.1 is beyond the scope of this paper and will be presented elsewhere.

Second, by (1.1)-(1.2), one can check that (2.1) is equivalent to the uniform positivity of the following (symmetric) matrix:

$$
\begin{aligned}
\mathcal{A} & \triangleq\left(\sum_{i^{\prime}, j^{\prime}}\left(a^{i j^{\prime}} a^{i^{\prime} j} d_{i^{\prime} j^{\prime}}+\frac{\left(a^{i j^{\prime}} a_{j^{\prime}}^{i^{\prime} j}+a^{j j^{\prime}} a_{j^{\prime}}^{i^{\prime} i}-a_{j^{\prime}}^{i j} a^{i^{\prime} j^{\prime}}\right) d_{i^{\prime}}}{2}\right)\right)_{1 \leq i, j \leq n} \\
& \equiv A \mathcal{H}_{d} A+\frac{1}{2}\left(\sum_{i^{\prime}, j^{\prime}}\left(a^{i j^{\prime}} a_{j^{\prime}}^{i^{\prime} j}+a^{j j^{\prime}} a_{j^{\prime}}^{i^{\prime} i}-a_{j^{\prime}}^{i j} a^{i^{\prime} j^{\prime}}\right) d_{i^{\prime}}\right)_{1 \leq i, j \leq n},
\end{aligned}
$$

where $\mathcal{H}_{d}$ is the Hessian matrix of $d(\cdot)$. Hence, if $A$ is a constant matrix, then $\mathcal{A}=A \mathcal{H}_{d} A$, and (2.1) is reduced to the (uniformly) strict convexity of $d(x)$. A little further, for any uniformly strict convex function $d(\cdot) \in C^{2}(\bar{\Omega})$, one can show that the matrix $A \mathcal{H}_{d} A$ is uniformly positive definite. Therefore, if

$$
\max _{1 \leq i, j, k \leq n} \sup _{x \in \bar{\Omega}}\left|a_{k}^{i j}(x)\right| \text { is small enough, }
$$

one concludes that $\mathcal{A}$ is uniformly positive definite. Consequently, if in addition, $d(\cdot)$ satisfies (2.2), then Condition 2.1 holds for $d(\cdot)$.

Third, the above remark, especially (2.4), does not mean that Condition 2.1 can hold only for coefficient matrices $A$ which are close to constant matrices. To illustrate this, let us state the following proposition, whose proof is presented in Appendix A.

Proposition 2.1. Let $n=2$, and let $A=\operatorname{diag}\left[a^{1}, a^{2}\right]$ with $a^{1} \in C^{2}(\bar{\Omega})$ and $a^{2} \in C^{1}(\bar{\Omega})$ being uniformly positive functions. Assume further that

(i) $a^{1}\left(x_{1}, x_{2}\right) \equiv a^{1}\left(x_{1}\right)$, i.e., it is independent of $x_{2}$;

(ii) $a_{1}^{1} a_{1}^{2} \geq 0$ in $\Omega$; and

(iii) there is at most one point $x_{1}^{0} \in G \triangleq \overline{\left\{x_{1} \in \mathbb{R} \mid\left(x_{1}, x_{2}\right) \in \Omega \text { for some } x_{2} \in \mathbb{R}\right\}}$ so that $a_{1}^{1}\left(x_{1}^{0}\right)=0$. Moreover, if such an $x_{1}^{0}$ exists, it satisfies $a_{11}^{1}\left(x_{1}^{0}\right)<0$. Then Condition 2.1 holds.

We emphasize that in the above, the derivatives $a_{1}^{1}(\cdot), a_{1}^{2}(\cdot)$, and $a_{2}^{2}(\cdot)$ are not necessarily small. Therefore, the matrix $A$ is not necessarily close to a constant matrix. 
As a more concrete case, let us look at the following situation: Let $a\left(x_{1}\right) \in C^{2}(G)$ be a uniformly positive and strictly concave function. One may check that if $a^{1}\left(x_{1}, x_{2}\right) \equiv$ $a^{2}\left(x_{1}, x_{2}\right) \equiv a\left(x_{1}\right)$, then $a^{1}$ and $a^{2}$ satisfy the conditions in Proposition 2.1. What is more interesting is that for this nonidentity matrix $A=a I$, if $a_{1}$, the derivative of $a$ with respect to $x_{1}$, changes sign, then one may further check that it does not satisfy the geometric condition introduced in [22, Theorem 2.2.4] (which, in our notation, reads $\frac{1}{2}-\left(x-x_{0}\right) \cdot \nabla a \geq 0$ in $\bar{\Omega}$, for some $\left.x_{0} \in \mathbb{R}^{n}\right)$ unless the length of $G$, or the positive part of $a_{1}$ in $G$ (i.e., $\max _{x \in G} a_{1}^{+}(x)$ ), or the negative part of $a_{1}$ in $G$ (i.e., $\max _{x \in G} a_{1}^{-}(x)$ ), is assumed to be sufficiently small. Hence, we have found a class of explicit and nontrivial examples satisfying our Condition 2.1. Also, we indicate that it is possible to construct nontrivial examples of nondiagonal coefficient matrices that satisfy Condition 2.1 .

For the function $d(\cdot)$ satisfying Condition 2.1, we introduce the following set:

$$
\Gamma_{+} \triangleq\left\{x \in \Gamma \mid \sum_{i, j} a^{i j} \nu_{i} d_{j}>0\right\},
$$

where $\nu=\nu(x)=\left(\nu_{1}, \nu_{2}, \ldots, \nu_{n}\right)$ is the unit outward normal vector of $\Omega$ at $x \in \Gamma$.

Note that for the case $A=I$, by choosing $d(x)=\left|x-x_{0}\right|^{2}$ with any given $x_{0} \in \mathbb{R}^{n} \backslash \bar{\Omega}$, we have Condition 2.1 with $\mu_{0}=4$, and (2.1) holds with an equality. In this case,

$$
\Gamma_{+}=\left\{x \in \Gamma \mid\left(x-x_{0}\right) \cdot \nu(x)>0\right\}
$$

which coincides with the usual star-shaped part of the whole boundary of $\Omega$ [29].

On the other hand, it is easy to check that, if $d(\cdot) \in C^{2}(\bar{\Omega})$ satisfies $(2.1)$, then for any given constants $a \geq 1$ and $b \in \mathbb{R}$, the function

$$
\hat{d}=\hat{d}(x) \triangleq a d(x)+b
$$

(scaling and translating $d(x)$ ) still satisfies Condition 2.1 with $\mu_{0}$ replaced by $a \mu_{0}$; meanwhile, the scaling and translating $d(x)$ do not change the set $\Gamma_{+}$. Hence, by scaling and translating $d(x)$, if necessary, we may assume without loss of generality that

$$
\left\{\begin{array}{l}
(2.1) \text { holds with } \mu_{0} \geq 4, \\
\frac{1}{4} \sum_{i, j} a^{i j}(x) d_{i}(x) d_{j}(x) \geq \max _{x \in \bar{\Omega}} d(x) \geq \min _{x \in \bar{\Omega}} d(x)>0 \quad \forall x \in \bar{\Omega} .
\end{array}\right.
$$

In what follows, we let

$$
R_{1} \triangleq \max _{x \in \bar{\Omega}} \sqrt{d(x)}, \quad T_{*} \triangleq 2 \inf \left\{R_{1} \mid d(\cdot) \text { satisfies }(2.7)\right\} .
$$

Concerning the controller $\omega$ in (1.5), we need the following assumption.

Condition 2.2. There is a constant $\delta>0$ such that

$$
\omega=\mathcal{O}_{\delta}\left(\Gamma_{+}\right) \bigcap \Omega
$$


Note that condition (2.9) can be replaced by

$$
\omega \supseteq \bar{\Gamma}_{+},
$$

which looks much weaker. In fact, when (2.10) holds, one can find a $\delta>0$ such that

$$
\omega \supseteq \mathcal{O}_{\delta}\left(\Gamma_{+}\right) \bigcap \Omega .
$$

It is not hard to see that if we can prove the controllability for (1.5) with a smaller controller $\omega$ satisfying (2.9), then we can do so for a larger controller $\omega$ satisfying (2.11) (in particular, we can choose $\omega$ to be $\mathcal{O}_{\delta}(\Gamma) \cap \Omega$, a neighborhood of the whole boundary $\Gamma$ ). We assume an equality in (2.9) only for simplicity of presentation.

The main controllability result in this paper is stated as follows.

TheOREM 2.2. Let $a^{i j}(\cdot) \in C^{1}(\bar{\Omega})$ satisfy $(1.1)-(1.2)$, and let $f(\cdot) \in C^{1}(\mathbb{R})$ satisfy (1.4). Let Conditions 2.1-2.2 hold. Then for any $T>T_{*}$, system (1.5) is exactly controllable in $H_{0}^{1}(\Omega) \times L^{2}(\Omega)$ at time $T$ by using some control $\gamma \in L^{2}((0, T) \times \omega)$.

In what follows, we will use $C$ to denote a generic positive constant which may vary from line to line (unless otherwise stated). As we mentioned before, the proof of Theorem 2.2 can be reduced to the following observability estimate result for system (1.7).

Theorem 2.3. Let $a^{i j}(\cdot) \in C^{1}(\bar{\Omega})$ satisfy $(1.1)-(1.2), q \in L^{\infty}\left(0, T ; L^{n}(\Omega)\right)$, and Conditions 2.1-2.2 hold. Then for any $T>T_{*}$, all weak solutions $w$ of system (1.7) satisfy estimate (1.8) with an observability constant $\mathcal{C}(q)>0$ of the form

$$
\mathcal{C}(q)=C \exp \left(C r^{2}\right)
$$

where

$$
r=|q|_{L^{\infty}\left(0, T ; L^{n}(\Omega)\right)} .
$$

Several remarks are in order.

Remark 2.1. By adopting the approach developed in this paper, Theorem 2.3 is strengthened in [8] as follows (see [8, Theorem 2.2]): Replace the assumption on $q$ by $q \in L^{\infty}\left(0, T ; L^{s}(\Omega)\right)$ for any fixed $s \in[n, \infty]$ and let the other assumptions in Theorem 2.3 remain unchanged. Then for any $T>T_{*}$, all weak solutions $w$ of system (1.7) satisfy estimate (1.8) with an observability constant $\mathcal{C}(q)>0$ of the form

$$
\mathcal{C}(q)=C \exp \left(C|q|_{L^{\infty}\left(0, T ; L^{s}(\Omega)\right)}^{\frac{1}{32-n / s}}\right) .
$$

On the other hand, it is shown in [8, Theorem 1.2] that the exponent $2 / 3$ in the estimate $|q|_{L^{\infty}\left(0, T ; L^{\infty}(\Omega)\right)}^{2 / 3}$ (in (2.14) for the special case $s=\infty$ ) is sharp. Although the problem of the optimality of the exponent $\frac{1}{3 / 2-n / s}$ in $|q|_{L^{\infty}\left(0, T ; L^{s}(\Omega)\right)}^{\frac{1}{3 / 2-n / s}}$ is unsolved when $s \in[n, \infty)$, [8, Theorem 1.2] does support the idea that the exponent 2 of the estimate $r^{2}$ in (2.12) might be sharp.

Remark 2.2. The "minimal" waiting time $T_{*}$ in Theorems $2.2-2.3$ is explicitly constructed (by (2.8)) but not sharp. The sharp $T_{*}$, as suggested by the special case $A=I$ considered in $[36,37]$, should be given as follows:

$$
\begin{aligned}
& T_{*} \triangleq 2 \inf \left\{R_{1} \mid d(x) \text { satisfies }(2.1) \text { with } \mu_{0} \geq 4\right. \text { and } \\
& \left.\qquad \frac{1}{4} \sum_{i, j} a^{i j}(x) d_{i}(x) d_{j}(x) \geq d(x) \geq \min _{x \in \bar{\Omega}} d(x)>0 \quad \forall x \in \bar{\Omega}\right\},
\end{aligned}
$$

Copyright $@$ by SIAM. Unauthorized reproduction of this article is prohibited. 
i.e., one replaces the term $\max _{x \in \bar{\Omega}} d(x)$ in (2.7) by $d(x)$. Unfortunately, we are unable to obtain such a sharp waiting time at this moment. One will see that the inequality involving $\sum_{i, j} a^{i j}(x) d_{i}(x) d_{j}(x)$ and $\max _{x \in \bar{\Omega}} d(x)$ in (2.7) plays a key role in (11.7).

Remark 2.3. Condition (1.4) on the nonlinearity $f(\cdot)$ in Theorem 2.2 is not sharp. As suggested in [45] for the one-dimensional problem, it is reasonable to expect that (1.4) may be relaxed to the following:

$$
\varlimsup_{s \rightarrow \infty} \frac{f(s)}{s \ln ^{2}|s|}=0 .
$$

But this remains unsolved for the time being.

Remark 2.4. Theorems 2.2-2.3 cover the main results in [26] except the minimal waiting time $T_{*}$.

Remark 2.5. Theorems 2.2 can be extended to the case when the nonlinearity $f(y)$ in (1.5) is replaced by $f(t, x, y)$, under suitable growth conditions on $(t, x, y)$. However, it seems to us that in the case when nonlinearity is $f\left(y, y_{t}, \nabla y\right)$, the technique developed in this paper is not enough, and one might have to employ the NashMoser-Hörmander iteration method [15] to overcome the difficulty due to the "loss of derivatives." The detailed study of this problem will be presented elsewhere. Note, however, that for purely PDE problems (existence and uniqueness of solutions, etc.) of the hyperbolic equations, the treatment on the nonlinearity $f\left(y, y_{t}, \nabla y\right)$ is almost the same as the simpler one, $f(y)$. This means that for the controllability problem of nonlinear systems, there exist some extra difficulties.

3. Some preliminaries. Let us consider the following linear inhomogeneous hyperbolic equation:

$$
\begin{cases}\mathcal{P} z=f & \text { in } Q \\ z=0 & \text { on } \Sigma\end{cases}
$$

In what follows, we call $z \in L^{2}(Q)$ a weak solution to (3.1) if

$$
(z, \mathcal{P} \eta)_{L^{2}(Q)}=\int_{0}^{T}\langle f(t, \cdot), \eta(t, \cdot)\rangle_{H^{-1}(\Omega), H_{0}^{1}(\Omega)} d t \quad \forall \eta \in C_{0}^{2}\left((0, T) ; H^{2}(\Omega) \cap H_{0}^{1}(\Omega)\right) .
$$

Note that in (3.1), no initial conditions are specified. Similarly to [40, Lemma 5.1], one can prove the following regularity result for system (3.1).

Lemma 3.1. Let $0<t_{1}<t_{2}<T, f \in L^{1}\left(0, T ; H^{-1}(\Omega)\right)$, and $g \in L^{2}\left(\left(t_{1}, t_{2}\right) \times \Omega\right)$ be given. Assume that $z \in L^{2}(Q)$ is a weak solution to (3.1), and $z=g$ in $\left(t_{1}, t_{2}\right) \times \Omega$. Then $z \in C\left([0, T] ; L^{2}(\Omega)\right) \cap C^{1}\left([0, T] ; H^{-1}(\Omega)\right)$, and there exists a constant $C>0$, depending only on $T, t_{1}, t_{2}, \Omega$, and $a^{i j}$, such that

$$
|z|_{C\left([0, T] ; L^{2}(\Omega)\right) \cap C^{1}\left([0, T] ; H^{-1}(\Omega)\right)} \leq C\left[|f|_{L^{1}\left(0, T ; H^{-1}(\Omega)\right)}+|g|_{L^{2}\left(\left(t_{1}, t_{2}\right) \times \Omega\right)}\right] .
$$

From the above, we see that $g$ plays the role of initial value for the weak solution $z$. Next, similarly to [36, Lemma 3.3] we have the following result.

Lemma 3.2. Let $a^{i j} \in C^{1}(\bar{\Omega})$ satisfy (1.1), and let $g \triangleq\left(g^{1}, \ldots, g^{n}\right): \mathbb{R}_{t} \times \mathbb{R}_{x}^{n} \rightarrow$ 
$\mathbb{R}^{n}$ be a vector field of class $C^{1}$. Then for any $z \in C^{2}\left(\mathbb{R}_{t} \times \mathbb{R}_{x}^{n}\right)$, we have

$$
\begin{aligned}
& -\sum_{j}\left[2(g \cdot \nabla z) \sum_{i} a^{i j} z_{i}+g^{j}\left(z_{t}^{2}-\sum_{i, k} a^{i k} z_{i} z_{k}\right)\right]_{j} \\
= & 2\left[(\mathcal{P} z) g \cdot \nabla z-\left(z_{t} g \cdot \nabla z\right)_{t}+z_{t} g_{t} \cdot \nabla z-\sum_{i, j, k} a^{i j} z_{i} z_{k} \frac{\partial g^{k}}{\partial x_{j}}\right] \\
& -(\nabla \cdot g) z_{t}^{2}+\sum_{i, j} z_{i} z_{j} \nabla \cdot\left(a^{i j} g\right) .
\end{aligned}
$$

Next, we denote the energy of system (1.7) by

$$
E(t) \triangleq \frac{1}{2}\left[\left|w_{t}(t, \cdot)\right|_{H^{-1}(\Omega)}^{2}+|w(t, \cdot)|_{L^{2}(\Omega)}^{2}\right] .
$$

Using the usual energy method, one obtains the following result.

Lemma 3.3. Let $T>0, q \in L^{\infty}\left(0, T ; L^{n}(\Omega)\right), w_{0} \in L^{2}(\Omega)$, and $w_{1} \in H^{-1}(\Omega)$. Then the weak solution $w(\cdot) \in C\left([0, T] ; L^{2}(\Omega)\right) \cap C^{1}\left([0, T] ; H^{-1}(\Omega)\right)$ of $(1.7)$ satisfies (recall (2.13) for $r$ )

$$
E(t) \leq C E(s) e^{C r} \quad \forall t, s \in[0, T] .
$$

Further, proceeding as in [36, Lemma 3.4], we conclude the following.

Lemma 3.4. Let $0 \leq S_{1}<S_{2}<T_{2}<T_{1} \leq T$ and $q \in L^{\infty}\left(0, T ; L^{n}(\Omega)\right)$. Then the weak solution $w(\cdot) \in C\left([0, T] ; L^{2}(\Omega)\right) \cap C^{1}\left([0, T] ; H^{-1}(\Omega)\right)$ of $(1.7)$ satisfies

$$
\int_{S_{2}}^{T_{2}} E(t) d t \leq C(1+r) \int_{S_{1}}^{T_{1}}|w(t, \cdot)|_{L^{2}(\Omega)}^{2} d t .
$$

Finally, the following proposition will be useful.

Proposition 3.5. For any $h>0, m=2,3, \ldots$, and $q_{m}^{i}, w_{m}^{i} \in \mathbb{C}(i=0,1, \ldots, m)$ with $q_{m}^{0}=q_{m}^{m}=0$, one has

$$
\begin{aligned}
-\sum_{i=1}^{m-1} q_{m}^{i} \frac{\left(w_{m}^{i+1}-2 w_{m}^{i}+w_{m}^{i-1}\right)}{h^{2}} & =\sum_{i=0}^{m-1} \frac{\left(q_{m}^{i+1}-q_{m}^{i}\right)}{h} \frac{\left(w_{m}^{i+1}-w_{m}^{i}\right)}{h} \\
& =\sum_{i=1}^{m} \frac{\left(q_{m}^{i}-q_{m}^{i-1}\right)}{h} \frac{\left(w_{m}^{i}-w_{m}^{i-1}\right)}{h} .
\end{aligned}
$$

Proof.

$$
\begin{aligned}
& -\sum_{i=1}^{m-1} q_{m}^{i} \frac{\left(w_{m}^{i+1}-2 w_{m}^{i}+w_{m}^{i-1}\right)}{h^{2}}=-\sum_{i=1}^{m-1} q_{m}^{i} \frac{\left(w_{m}^{i+1}-w_{m}^{i}\right)}{h^{2}}+\sum_{i=1}^{m-1} q_{m}^{i} \frac{\left(w_{m}^{i}-w_{m}^{i-1}\right)}{h^{2}} \\
& =\sum_{i=1}^{m-1} \frac{\left(q_{m}^{i+1}-q_{m}^{i}\right)}{h} \frac{\left(w_{m}^{i+1}-w_{m}^{i}\right)}{h}-\sum_{i=1}^{m-1} \frac{q_{m}^{i+1}}{h} \frac{\left(w_{m}^{i+1}-w_{m}^{i}\right)}{h}+\sum_{i=0}^{m-2} \frac{q_{m}^{i+1}}{h} \frac{\left(w_{m}^{i+1}-w_{m}^{i}\right)}{h} \\
& =\sum_{i=1}^{m-1} \frac{\left(q_{m}^{i+1}-q_{m}^{i}\right)}{h} \frac{\left(w_{m}^{i+1}-w_{m}^{i}\right)}{h}+\frac{q_{m}^{1}}{h} \frac{\left(w_{m}^{1}-w_{m}^{0}\right)}{h} \\
& =\sum_{i=0}^{m-1} \frac{\left(q_{m}^{i+1}-q_{m}^{i}\right)}{h} \frac{\left(w_{m}^{i+1}-w_{m}^{i}\right)}{h},
\end{aligned}
$$

which gives the desired equality. 
4. Second order differential operators with symmetric coefficients. In this section, we consider second order differential operators with symmetric coefficients. Our hyperbolic differential operator $\mathcal{P}$ is of such a type. We will establish a pointwise equality and a couple of inequalities for such differential operators, which will play important roles. First, we have the following identity.

THEOREM 4.1. Let $m \in \mathbb{N}$,

$$
b^{i j}=b^{j i} \in C^{1}\left(\mathbb{R}^{m}\right), \quad i, j=1,2, \ldots, m,
$$

and $u, \ell, \Psi \in C^{2}\left(\mathbb{R}^{m}\right)$. Set $\theta=e^{\ell}$ and $v=\theta u$. Then

$$
\begin{gathered}
\theta^{2}\left|\sum_{i, j}\left(b^{i j} u_{i}\right)_{j}\right|^{2}+2 \sum_{j}\left\{2 \sum_{i, i^{\prime}, j^{\prime}} b^{i j} b^{i^{\prime} j^{\prime}} \ell_{i^{\prime}} v_{i} v_{j^{\prime}}-\sum_{i, i^{\prime}, j^{\prime}} b^{i j} b^{i^{\prime} j^{\prime}} \ell_{i} v_{i^{\prime}} v_{j^{\prime}}\right. \\
\left.+\Psi \sum_{i} b^{i j} v_{i} v-\sum_{i} b^{i j}\left[(\Lambda+\Psi) \ell_{i}+\frac{\Psi_{i}}{2}\right] v^{2}\right\}_{j} \\
=2 \sum_{i, j}\left\{\sum_{i^{\prime}, j^{\prime}}\left[2 b^{i j^{\prime}}\left(b^{i^{\prime} j} \ell_{i^{\prime}}\right)_{j^{\prime}}-\left(b^{i j} b^{i^{\prime} j^{\prime}} \ell_{i^{\prime}}\right)_{j^{\prime}}\right]+\Psi b^{i j}\right\} v_{i} v_{j}+B v^{2} \\
+\left|\sum_{i, j}\left(b^{i j} v_{i}\right)_{j}-\Lambda v\right|^{2}+4\left|\sum_{i, j} b^{i j} \ell_{i} v_{j}\right|^{2},
\end{gathered}
$$

where

$$
\left\{\begin{array}{l}
\Lambda \triangleq-\sum_{i, j}\left(b^{i j} \ell_{i} \ell_{j}-b_{j}^{i j} \ell_{i}-b^{i j} \ell_{i j}\right)-\Psi \\
B \triangleq 2\left[\Lambda \Psi-\sum_{i, j}\left((\Lambda+\Psi) b^{i j} \ell_{i}\right)_{j}\right]+\Psi^{2}-\sum_{i, j}\left(b^{i j} \Psi_{j}\right)_{i} .
\end{array}\right.
$$

We see that only the symmetry condition (4.1) is assumed in the above. Hence, Theorem 4.1 is applicable to hyperbolic and ultrahyperbolic operators.

Theorem 4.1 looks similar to [25, Lemma 1, p. 124] (which is devoted to a similar problem for a class of ultrahyperbolic operators). The main difference is that we leave the function $v$ on the right-hand side of (4.2) without returning to $u$, unlike the result of [25] mentioned above, which has only the variable $u$ on both sides. Our result greatly simplifies the computation. Also, a similar idea played a key role in establishing the observability estimate for the wave equations with Neumann boundary conditions in [24] (which should be compared with [19]). We refer the reader to $[12,13]$ for further application of Theorem 4.1 and its generalization, and to $[7]$ for related work.

Proof of Theorem 4.1. The proof is divided into several steps.

Step 1. Recalling $\theta=e^{\ell}$ and $v=\theta u$, one has $u_{i}=\theta^{-1}\left(v_{i}-\ell_{i} v\right)(i=1,2, \ldots, m)$. By the symmetry condition (4.1), it is easy to see that

$$
\sum_{i, j} b^{i j}\left(\ell_{i} v_{j}+\ell_{j} v_{i}\right)=2 \sum_{i, j} b^{i j} \ell_{i} v_{j}
$$

Copyright (c) by SIAM. Unauthorized reproduction of this article is prohibited. 
Thus, we obtain

$$
\begin{aligned}
& \sum_{i, j}\left(b^{i j} u_{i}\right)_{j}=\sum_{i, j}\left[\theta^{-1} b^{i j}\left(v_{i}-\ell_{i} v\right)\right]_{j} \\
& =\theta^{-1} \sum_{i, j}\left[b^{i j}\left(v_{i}-\ell_{i} v\right)\right]_{j}-\theta^{-1} \sum_{i, j} b^{i j}\left(v_{i}-\ell_{i} v\right) \ell_{j} \\
& =\theta^{-1} \sum_{i, j}\left[\left(b^{i j} v_{i}\right)_{j}-b^{i j}\left(\ell_{i} v_{j}+\ell_{j} v_{i}\right)+\left(b^{i j} \ell_{i} \ell_{j}-b_{j}^{i j} \ell_{i}-b^{i j} \ell_{i j}\right) v\right] \\
& =\theta^{-1} \sum_{i, j}\left[\left(b^{i j} v_{i}\right)_{j}-2 b^{i j} \ell_{i} v_{j}+\left(b^{i j} \ell_{i} \ell_{j}-b_{j}^{i j} \ell_{i}-b^{i j} \ell_{i j}\right) v\right] \\
& \equiv-\theta^{-1}\left(I_{1}+I_{2}+I_{3}\right),
\end{aligned}
$$

where

$$
\left\{\begin{aligned}
I_{1} & \triangleq-\sum_{i, j}\left[\left(b^{i j} v_{i}\right)_{j}+\left(b^{i j} \ell_{i} \ell_{j}-b_{j}^{i j} \ell_{i}-b^{i j} \ell_{i j}\right) v\right]-\Psi v \\
& =-\sum_{i, j}\left(b^{i j} v_{i}\right)_{j}+\Lambda v \\
I_{2} & \triangleq 2 \sum_{i, j} b^{i j} \ell_{i} v_{j}, \quad I_{3} \triangleq \Psi v .
\end{aligned}\right.
$$

Then, by (4.4) and (4.5), we get

$$
\theta^{2}\left|\sum_{i, j}\left(b^{i j} u_{i}\right)_{j}\right|^{2}=\left|I_{1}\right|^{2}+\left|I_{2}\right|^{2}+\left|I_{3}\right|^{2}+2\left(I_{1} I_{2}+I_{2} I_{3}+I_{1} I_{3}\right) .
$$

Step 2. Let us compute $I_{1} I_{2}$. Using (4.1) again, and noting

$$
\sum_{i, j, i^{\prime}, j^{\prime}}\left(b^{i j} b^{i^{\prime} j^{\prime}} \ell_{i^{\prime}} v_{i} v_{j}\right)_{j^{\prime}}=\sum_{i, j, i^{\prime}, j^{\prime}}\left(b^{i j} b^{i^{\prime} j^{\prime}} \ell_{i} v_{i^{\prime}} v_{j^{\prime}}\right)_{j}
$$

we get

$$
\begin{aligned}
& 2 \sum_{i, j, i^{\prime}, j^{\prime}} b^{i j} b^{i^{\prime} j^{\prime}} \ell_{i^{\prime}} v_{i} v_{j j^{\prime}} \\
& =\sum_{i, j, i^{\prime}, j^{\prime}} b^{i j} b^{i^{\prime} j^{\prime}} \ell_{i^{\prime}}\left(v_{i} v_{j j^{\prime}}+v_{j} v_{i j^{\prime}}\right)=\sum_{i, j, i^{\prime}, j^{\prime}} b^{i j} b^{i^{\prime} j^{\prime}} \ell_{i^{\prime}}\left(v_{i} v_{j}\right)_{j^{\prime}} \\
& =\sum_{i, j, i^{\prime}, j^{\prime}}\left(b^{i j} b^{i^{\prime} j^{\prime}} \ell_{i^{\prime}} v_{i} v_{j}\right)_{j^{\prime}}-\sum_{i, j, i^{\prime}, j^{\prime}}\left(b^{i j} b^{i^{\prime} j^{\prime}} \ell_{i^{\prime}}\right)_{j^{\prime}} v_{i} v_{j} \\
& =\sum_{i, j, i^{\prime}, j^{\prime}}\left(b^{i j} b^{i^{\prime} j^{\prime}} \ell_{i} v_{i^{\prime}} v_{j^{\prime}}\right)_{j}-\sum_{i, j, i^{\prime}, j^{\prime}}\left(b^{i j} b^{i^{\prime} j^{\prime}} \ell_{i^{\prime}}\right)_{j^{\prime}} v_{i} v_{j} .
\end{aligned}
$$

Hence, by (4.5) and (4.7), and noting

$$
\sum_{i, j, i^{\prime}, j^{\prime}} b^{i j}\left(b^{i^{\prime} j^{\prime}} \ell_{i^{\prime}}\right)_{j} v_{i} v_{j^{\prime}}=\sum_{i, j, i^{\prime}, j^{\prime}} b^{i j^{\prime}}\left(b^{i^{\prime} j} \ell_{i^{\prime}}\right)_{j^{\prime}} v_{i} v_{j},
$$

Copyright $@$ by SIAM. Unauthorized reproduction of this article is prohibited. 
we get

$$
\begin{aligned}
I_{1} I_{2}= & 2 \sum_{i, j} b^{i j} \ell_{i} v_{j}\left[-\sum_{i, j}\left(b^{i j} v_{i}\right)_{j}+\Lambda v\right] \\
= & -2 \sum_{i, j, i^{\prime}, j^{\prime}}\left(b^{i j} b^{i^{\prime} j^{\prime}} \ell_{i^{\prime}} v_{i} v_{j^{\prime}}\right)_{j}+2 \sum_{i, j, i^{\prime}, j^{\prime}} b^{i j}\left(b^{i^{\prime} j^{\prime}} \ell_{i^{\prime}}\right)_{j} v_{i} v_{j^{\prime}} \\
& +2 \sum_{i, j, i^{\prime}, j^{\prime}} b^{i j} b^{i^{\prime} j^{\prime}} \ell_{i^{\prime}} v_{i} v_{j^{\prime} j}+\Lambda \sum_{i, j} b^{i j} \ell_{i}\left(v^{2}\right)_{j} \\
= & -\sum_{j}\left(2 \sum_{i, i^{\prime}, j^{\prime}} b^{i j} b^{i^{\prime} j^{\prime}} \ell_{i^{\prime}} v_{i} v_{j^{\prime}}-\sum_{i, i^{\prime}, j^{\prime}} b^{i j} b^{i^{\prime} j^{\prime}} \ell_{i} v_{i^{\prime}} v_{j^{\prime}}-\Lambda \sum_{i} b^{i j} \ell_{i} v^{2}\right)_{j} \\
& +\sum_{i, j, i^{\prime}, j^{\prime}}\left[2 b^{i j^{\prime}}\left(b^{i^{\prime} j} \ell_{i^{\prime}}\right)_{j^{\prime}}-\left(b^{i j} b^{i^{\prime} j^{\prime}} \ell_{i^{\prime}}\right)_{j^{\prime}}\right] v_{i} v_{j}-\sum_{i, j}\left(\Lambda b^{i j} \ell_{i}\right)_{j} v^{2} .
\end{aligned}
$$

Step 3. Let us compute $I_{2} I_{3}$ and $I_{1} I_{3}$. By (4.5), we see that

$$
\begin{aligned}
I_{2} I_{3} & =2 \Psi v \sum_{i, j} b^{i j} \ell_{i} v_{j}=\Psi \sum_{i, j} b^{i j} \ell_{i}\left(v^{2}\right)_{j} \\
& =\sum_{i, j}\left(\Psi b^{i j} \ell_{i} v^{2}\right)_{j}-\sum_{i, j}\left(\Psi b^{i j} \ell_{i}\right)_{j} v^{2} .
\end{aligned}
$$

Similarly, by (4.5), we get

$$
\begin{aligned}
2 I_{1} I_{3} & =2 \Psi v\left[-\sum_{i, j}\left(b^{i j} v_{i}\right)_{j}+\Lambda v\right] \\
& =-2 \sum_{i, j}\left(\Psi b^{i j} v v_{i}\right)_{j}+2 \Psi \sum_{i, j} b^{i j} v_{i} v_{j}+\sum_{i, j} b^{i j} \Psi_{j}\left(v^{2}\right)_{i}+2 \Lambda \Psi v^{2} \\
& =-\sum_{i, j}\left(2 \Psi b^{i j} v v_{i}-b^{i j} \Psi_{i} v^{2}\right)_{j}+2 \Psi \sum_{i, j} b^{i j} v_{i} v_{j}+\left[-\sum_{i, j}\left(b^{i j} \Psi_{j}\right)_{i}+2 \Lambda \Psi\right] v^{2} .
\end{aligned}
$$

Step 4. Finally, combining (4.6), (4.8), (4.9), and (4.10), we immediately conclude with the desired equality (4.2). This completes the proof of Theorem 4.1.

As a consequence of Theorem 4.1, we have the following.

Corollary 4.2. Let $a^{i j} \in C^{1}(\bar{\Omega})$ satisfy (1.1), and let $u, \ell, \Psi \in C^{2}\left(\mathbb{R}^{1+n}\right)$. Let $\theta=e^{\ell}$ and $v=\theta u$. Then

$$
\begin{aligned}
& \theta^{2}|\mathcal{P} u|^{2} \\
& +2\left[\ell_{t}\left(v_{t}^{2}+\sum_{i, j} a^{i j} v_{i} v_{j}\right)-2 \sum_{i, j} a^{i j} \ell_{i} v_{j} v_{t}-\Psi v v_{t}+\left((\Lambda+\Psi) \ell_{t}+\frac{\Psi_{t}}{2}\right) v^{2}\right]_{t} \\
& +2 \sum_{j}\left\{2 \sum_{i, i^{\prime}, j^{\prime}} a^{i j} a^{i^{\prime} j^{\prime}} \ell_{i^{\prime}} v_{i} v_{j^{\prime}}-\sum_{i, i^{\prime}, j^{\prime}} a^{i j} a^{i^{\prime} j^{\prime}} \ell_{i} v_{i^{\prime}} v_{j^{\prime}}+\Psi v \sum_{i} a^{i j} v_{i}\right. \\
& \left.-2 \ell_{t} v_{t} \sum_{i} a^{i j} v_{i}+\sum_{i} a^{i j} \ell_{i} v_{t}^{2}-\sum_{i} a^{i j}\left[(\Lambda+\Psi) \ell_{i}+\frac{\Psi_{i}}{2}\right] v^{2}\right\}_{j} \\
& \geq 2\left(\ell_{t t}+\sum_{i, j}\left(a^{i j} \ell_{i}\right)_{j}-\Psi\right) v_{t}^{2}-8 \sum_{i, j} a^{i j} \ell_{j t} v_{i} v_{t} \\
& +2 \sum_{i, j}\left\{a^{i j} \ell_{t t}+\sum_{i^{\prime}, j^{\prime}}\left[2 a^{i j^{\prime}}\left(a^{i^{\prime} j} \ell_{i^{\prime}}\right)_{j^{\prime}}-\left(a^{i j} a^{i^{\prime} j^{\prime}} \ell_{i^{\prime}}\right)_{j^{\prime}}\right]+\Psi a^{i j}\right\} v_{i} v_{j}+B v^{2},
\end{aligned}
$$

Copyright $@$ by SIAM. Unauthorized reproduction of this article is prohibited. 
where

$$
\left\{\begin{aligned}
\Lambda=\left(\ell_{t}^{2}-\ell_{t t}\right)-\sum_{i, j}\left(a^{i j} \ell_{i} \ell_{j}-a_{j}^{i j} \ell_{i}-a^{i j} \ell_{i j}\right)-\Psi, \\
B=2\left[\Lambda \Psi+\left((\Lambda+\Psi) \ell_{t}\right)_{t}-\sum_{i, j}\left((\Lambda+\Psi) a^{i j} \ell_{i}\right)_{j}\right] \\
+\Psi^{2}+\Psi_{t t}-\sum_{i, j}\left(a^{i j} \Psi_{j}\right)_{i} .
\end{aligned}\right.
$$

In particular, if

$$
\left\{\begin{array}{l}
\phi=\phi(t, x) \triangleq d(x)-c(t-T / 2)^{2} \\
\Psi \triangleq \lambda\left[\sum_{i, j}\left(a^{i j} d_{i}\right)_{j}-2 c-1+k\right] \\
\ell \triangleq \lambda \phi, \quad v \triangleq \theta u, \quad \theta \triangleq e^{\ell}
\end{array}\right.
$$

with $\lambda, T>0, c \in(0,1)$, and $k \in \mathbb{R}$, then

$$
\text { (left-hand side of }(4.11)) \geq 2 \lambda(1-k) v_{t}^{2}
$$

$$
+2 \lambda \sum_{i, j}\left\{(k-1-4 c) a^{i j}+\sum_{i^{\prime}, j^{\prime}}\left[2 a^{i j^{\prime}}\left(a^{i^{\prime} j} d_{i^{\prime}}\right)_{j^{\prime}}-a_{j^{\prime}}^{i j} a^{i^{\prime} j^{\prime}} d_{i^{\prime}}\right]\right\} v_{i} v_{j}+B v^{2}
$$

where

$$
\left\{\begin{array}{c}
\Lambda=\lambda^{2}\left[4 c^{2}(t-T / 2)^{2}-\sum_{i, j} a^{i j} d_{i} d_{j}\right]+\lambda(4 c+1-k), \\
B=2 \lambda^{3}\left[(4 c+1-k) \sum_{i, j} a^{i j} d_{i} d_{j}+\sum_{i, j} a^{i j} d_{i}\left(\sum_{i^{\prime}, j^{\prime}} a^{i^{\prime} j^{\prime}} d_{i^{\prime}} d_{j^{\prime}}\right)_{j}\right. \\
\left.-4(8 c+1-k) c^{2}(t-T / 2)^{2}\right]+O\left(\lambda^{2}\right) .
\end{array}\right.
$$

Proof. Using Theorem 4.1 with $m=1+n$, and

$$
\left(b^{i j}\right)_{m \times m}=\left(\begin{array}{cc}
-1 & 0 \\
0 & A
\end{array}\right),
$$

by a direct calculation, we obtain (4.11). The inequality occurs because we have dropped the last two nonnegative terms (see (4.2)). Next, by the choice of (4.13), we can obtain (4.14).

Copyright (c) by SIAM. Unauthorized reproduction of this article is prohibited. 
5. Global Carleman estimate for the hyperbolic operators in $H_{0}^{1}(Q)$. Recall (2.8) for the definitions of $R_{1}$ and $T_{*}$. Let $T>T_{*}$ be given. We may assume that

$$
T>2 R_{1} .
$$

By (5.1), one may choose a constant $c \in(0,1)$ so that

$$
\left(\frac{2 R_{1}}{T}\right)^{2}<c<\frac{2 R_{1}}{T}
$$

Henceforth, we choose $\phi(t, x)$ as in (4.13) with $T$ and $c$ satisfying, respectively, (5.1) and (5.2).

Remark 5.1. The function $\phi$ constructed above, together with Condition 2.1, will play a similar role in establishing the Carleman estimate for the hyperbolic operators to that of the function $\psi$ in [18, Condition 1.1], both of which are pseudoconvex in the sense of [17, Definition 28.3.1]. We refer to the classical monographs [16, 17] for more extensive treatment of the Carleman estimate for general partial differential operators, based on pseudoconvex assumptions. Note, however, that our more concrete and explicit choice of $\phi$ has the following advantages:

(1) It avoids the complicated verification of the pseudoconvex assumption, say, Condition 1.1 in [18]. Indeed, we need only check the "convexity" condition (2.1) and the nonvanishing condition (2.2) (see Proposition 2.1 for an example).

(2) Our $\phi$ is more natural. In this respect, we note that the time variable $t$ and spatial variables $x$ are separate, which matches the very fact that for the principal operator $\mathcal{P}$, the time derivative $\partial_{t t}$ and the spatial derivatives $-\sum_{i, j} \partial_{j}\left(a^{i j}(x) \partial_{i}\right)$ have a similar separation property.

(3) The explicit form of $\phi(\cdot)$ or $d(\cdot)$ is useful in the definition of the "controlled/observed" subboundary $\Gamma_{+}$in (2.5). Also, it plays a key role by scaling and translating $d(x)$ as in (2.6) to achieve (2.7).

(4) What is more important, as mentioned before, is that with our assumption of Condition 2.1, we can give an explicit formula for the waiting time $T_{*}$, but this seems to be impossible in the setting of [18] and in that of $[16,17]$. Meanwhile, as we shall see later (in the proof of Theorem 2.3), the explicit form of $\phi(\cdot)$ will play a crucial role in deducing the key estimate $(2.12)$ on the observability constant $\mathcal{C}(q)$.

The following Carleman estimate will play a crucial role in section 7 .

TheOrem 5.1. Let $a^{i j} \in C^{1}(\bar{\Omega})$ satisfy (1.1)-(1.2), and let Conditions 2.1-2.2 hold. Then there exists a $\lambda_{0}>1$ such that for all $\lambda \geq \lambda_{0}$ and all $u \in H_{0}^{1}(Q)$ with $\mathcal{P} u \in L^{2}(Q)$, it holds that

$$
\begin{aligned}
& \lambda \int_{Q}\left(\lambda^{2} u^{2}+u_{t}^{2}+|\nabla u|^{2}\right) e^{2 \lambda \phi} d x d t \\
& \leq C\left[\left|e^{\lambda \phi} \mathcal{P} u\right|_{L^{2}(Q)}^{2}+\lambda^{2} \int_{0}^{T} \int_{\omega}\left(\lambda^{2} u^{2}+u_{t}^{2}\right) e^{2 \lambda \phi} d x d t\right] .
\end{aligned}
$$

For the reader's convenience, in Appendix B we will give a proof of Theorem 5.1 which is close to the spirit of [24].

Remark 5.2. In the above theorem, the main element, which enables one to integrate over the entire cylinder $Q$ instead of the "conventional" case of its subdomain 
bounded by a level surface of the function $\phi$, is that $u(0, x)=u(T, x)=0$ in $\Omega$. From the proof of Theorem 5.1, one can see that this point is achieved via (11.10). In the cases $A=I$, and more generally $A=a(x) I$, with a quite restrictive positive function $a(x)$, inequality (11.10) actually follows from [22, equation (2.2.51)] if we introduce (in this paper) a new variable $\tau=t-T / 2$ instead of the time variable $t$.

6. An auxiliary optimal control problem. In this section, we will present an auxiliary optimal control problem which will be useful later. Although some ideas are taken from [18, pp. 190-199], our presentation seems to be easier to understand.

Throughout this section, we fix $\phi$ as in (4.13), a parameter $\lambda>0$, and a function $u \in C\left([0, T] ; L^{2}(\Omega)\right)$ satisfying $u(0, x)=u(T, x)=0$ for $x \in \Omega$. For any $K>1$, we choose a function $\varrho \equiv \varrho^{K}(x) \in C^{2}(\bar{\Omega})$ with $\min _{x \in \bar{\Omega}} \varrho(x)=1$ so that (recall Condition 2.2 for $\omega)$

$$
\varrho(x)= \begin{cases}1 & \text { for } x \in \omega, \\ K & \text { for } \operatorname{dist}(x, \omega) \geq \frac{1}{\ln K} .\end{cases}
$$

Next, fix any integer $m \geq 3$. Let $h=\frac{T}{m}$. Define

$$
u_{m}^{i} \equiv u_{m}^{i}(x)=u(i h, x), \quad \phi_{m}^{i} \equiv \phi_{m}^{i}(x)=\phi(i h, x), \quad i=0,1, \ldots, m .
$$

Let $\left\{\left(z_{m}^{i}, r_{1 m}^{i}, r_{2 m}^{i}, r_{m}^{i}\right)\right\}_{i=0}^{m} \in\left(H_{0}^{1}(\Omega) \times\left(L^{2}(\Omega)\right)^{3}\right)^{m+1}$ satisfy the following system:

$$
\begin{cases}\frac{z_{m}^{i+1}-2 z_{m}^{i}+z_{m}^{i-1}}{h^{2}}-\sum_{j_{1}, j_{2}=1}^{n} \partial_{x_{j_{2}}}\left(a^{j_{1} j_{2}} \partial_{x_{j_{1}}} z_{m}^{i}\right) & \\ =\frac{r_{1 m}^{i+1}-r_{1 m}^{i}}{h}+r_{2 m}^{i}+\lambda u_{m}^{i} e^{2 \lambda \phi_{m}^{i}}+r_{m}^{i}, \quad(1 \leq i \leq m-1) & \text { in } \Omega, \\ z_{m}^{i}=0, \quad(0 \leq i \leq m) & \text { on } \Gamma \\ z_{m}^{0}=z_{m}^{m}=r_{2 m}^{0}=r_{2 m}^{m}=r_{m}^{0}=r_{m}^{m}=0, \quad r_{1 m}^{0}=r_{1 m}^{1} & \text { in } \Omega .\end{cases}
$$

Note that we do not assume $r_{1 m}^{0}$ and $r_{1 m}^{m}$ vanish; instead we assume $r_{1 m}^{0}=r_{1 m}^{1}$. In system (6.3), $\left(r_{1 m}^{i}, r_{2 m}^{i}, r_{m}^{i}\right) \in\left(L^{2}(\Omega)\right)^{3}(i=0,1, \ldots, m)$ can be regarded as controls. The set of admissible sequences for (6.3) is defined as

$$
\begin{aligned}
\mathcal{A}_{a d} \triangleq\left\{\left\{\left(z_{m}^{i}, r_{1 m}^{i}, r_{2 m}^{i}, r_{m}^{i}\right)\right\}_{i=0}^{m} \in\left(H_{0}^{1}(\Omega) \times\left(L^{2}(\Omega)\right)^{3}\right)^{m+1} \mid\right. \\
\left.\left\{\left(z_{m}^{i}, r_{1 m}^{i}, r_{2 m}^{i}, r_{m}^{i}\right)\right\}_{i=0}^{m} \text { satisfy }(6.3)\right\} .
\end{aligned}
$$

Since $\left\{\left(0,0,0,-\lambda u_{m}^{i} e^{2 \lambda \phi_{m}^{i}}\right)\right\}_{i=0}^{m} \in \mathcal{A}_{a d}$, one sees that $\mathcal{A}_{a d} \neq \emptyset$.

Next, let us introduce the cost functional

$$
\begin{aligned}
& J\left(\left\{\left(z_{m}^{i}, r_{1 m}^{i}, r_{2 m}^{i}, r_{m}^{i}\right)\right\}_{i=0}^{m}\right) \\
& =\frac{h}{2} \int_{\Omega} \varrho \frac{\left|r_{1 m}^{m}\right|^{2}}{\lambda^{2}} e^{-2 \lambda \phi_{m}^{m}} d x \\
& \quad+\frac{h}{2} \sum_{i=1}^{m-1}\left[\int_{\Omega}\left|z_{m}^{i}\right|^{2} e^{-2 \lambda \phi_{m}^{i}} d x+\int_{\Omega} \varrho\left(\frac{\left|r_{1 m}^{i}\right|^{2}}{\lambda^{2}}+\frac{\left|r_{2 m}^{i}\right|^{2}}{\lambda^{4}}\right) e^{-2 \lambda \phi_{m}^{i}} d x+K \int_{\Omega}\left|r_{m}^{i}\right|^{2} d x\right] .
\end{aligned}
$$


We pose the following optimal control problem: Find a $\left\{\left(\hat{z}_{m}^{i}, \hat{r}_{1 m}^{i}, \hat{r}_{2 m}^{i}, \hat{r}_{m}^{i}\right)\right\}_{i=0}^{m} \in \mathcal{A}_{a d}$ such that

$$
\begin{aligned}
& J\left(\left\{\left(\hat{z}_{m}^{i}, \hat{r}_{1 m}^{i}, \hat{r}_{2 m}^{i}, \hat{r}_{m}^{i}\right)\right\}_{i=0}^{m}\right) \\
& =\min _{\left\{\left(z_{m}^{i}, r_{1 m}^{i}, r_{2 m}^{i}, r_{m}^{i}\right)\right\}_{i=0}^{m} \in \mathcal{A}_{a d}} J\left(\left\{\left(z_{m}^{i}, r_{1 m}^{i}, r_{2 m}^{i}, r_{m}^{i}\right)\right\}_{i=0}^{m}\right) .
\end{aligned}
$$

Note that for any $\left\{\left(z_{m}^{i}, r_{1 m}^{i}, r_{2 m}^{i}, r_{m}^{i}\right)\right\}_{i=0}^{m} \in \mathcal{A}_{a d}$, by standard regularity results of elliptic equations, one has that $z_{m}^{i} \in H^{2}(\Omega) \cap H_{0}^{1}(\Omega)$. The following technical result will play a crucial role in section 7 .

Proposition 6.1. For any $K>1$ and $m \geq 3$, problem (6.5) admits a unique solution $\left\{\left(\hat{z}_{m}^{i}, \hat{r}_{1 m}^{i}, \hat{r}_{2 m}^{i}, \hat{r}_{m}^{i}\right)\right\}_{i=0}^{m} \in \mathcal{A}_{a d}$ (which depends on $\left.K\right)$. Furthermore, for

$$
p_{m}^{i} \equiv p_{m}^{i}(x) \triangleq K \hat{r}_{m}^{i}(x), \quad 0 \leq i \leq m,
$$

one has

$$
\hat{z}_{m}^{0}=\hat{z}_{m}^{m}=p_{m}^{0}=p_{m}^{m}=0 \text { in } \Omega, \hat{z}_{m}^{i}, p_{m}^{i} \in H^{2}(\Omega) \cap H_{0}^{1}(\Omega) \text { for } 1 \leq i \leq m-1,
$$

and the following optimality conditions hold:

$$
\left\{\begin{array}{ll}
\frac{p_{m}^{i}-p_{m}^{i-1}}{h}+\varrho \frac{\hat{r}_{1 m}^{i}}{\lambda^{2}} e^{-2 \lambda \phi_{m}^{i}}=0 & \text { in } \Omega, \\
p_{m}^{i}-\varrho \frac{\hat{r}_{2 m}^{i}}{\lambda^{4}} e^{-2 \lambda \phi_{m}^{i}}=0 & \text { in } \Omega,
\end{array} \quad 1 \leq i \leq m,\right.
$$

$$
\left\{\begin{array}{cc}
\frac{p_{m}^{i+1}-2 p_{m}^{i}+p_{m}^{i-1}}{h^{2}}-\sum_{j_{1}, j_{2}=1}^{n} \partial_{x_{j_{2}}}\left(a^{j_{1} j_{2}} \partial_{x_{j_{1}}} p_{m}^{i}\right)+\hat{z}_{m}^{i} e^{-2 \lambda \phi_{m}^{i}}=0 & \text { in } \Omega \\
p_{m}^{i}=0 & \text { on } \Gamma \\
1 \leq i \leq m-1 . &
\end{array}\right.
$$

Moreover, there is a constant $C=C(K, \lambda)>0$, independent of $m$, such that

$$
h \sum_{i=1}^{m-1} \int_{\Omega}\left[\left|\hat{z}_{m}^{i}\right|^{2}+\left|\hat{r}_{1 m}^{i}\right|^{2}+\left|\hat{r}_{2 m}^{i}\right|^{2}+K\left|\hat{r}_{m}^{i}\right|^{2}\right] d x+h \int_{\Omega}\left|\hat{r}_{1 m}^{m}\right|^{2} d x \leq C,
$$

and

$$
\begin{aligned}
h \sum_{i=0}^{m-1} \int_{\Omega}\left[\frac{\left(\hat{z}_{m}^{i+1}-\hat{z}_{m}^{i}\right)^{2}}{h^{2}}+\frac{\left(\hat{r}_{1 m}^{i+1}-\hat{r}_{1 m}^{i}\right)^{2}}{h^{2}}\right. & +\frac{\left(\hat{r}_{2 m}^{i+1}-\hat{r}_{2 m}^{i}\right)^{2}}{h^{2}} \\
& \left.+K \frac{\left(\hat{r}_{m}^{i+1}-\hat{r}_{m}^{i}\right)^{2}}{h^{2}}\right] d x \leq C .
\end{aligned}
$$

We refer to Appendix C for a proof of this proposition.

7. Global Carleman estimate for hyperbolic operators in $L^{2}(Q)$. In order to prove Theorem 2.3, we need the following result.

Copyright $@$ by SIAM. Unauthorized reproduction of this article is prohibited. 
Theorem 7.1. Let $a^{i j} \in C^{1}(\bar{\Omega})$ satisfy (1.1)-(1.2). Let Conditions 2.1-2.2 hold. Then for any $\lambda \geq \lambda_{0} \geq 1$, and any $u \in C\left([0, T] ; L^{2}(\Omega)\right)$ satisfying $u(0, x)=u(T, x)=$ 0 for $x \in \Omega, \mathcal{P} u \in H^{-1}(Q)$, and

$$
(u, \mathcal{P} \eta)_{L^{2}(Q)}=\langle\mathcal{P} u, \eta\rangle_{H^{-1}(Q), H_{0}^{1}(Q)} \quad \forall \eta \in H_{0}^{1}(Q) \text { with } \mathcal{P} \eta \in L^{2}(Q),
$$

it holds that

$$
\lambda \int_{Q} u^{2} e^{2 \lambda \phi} d x d t \leq C\left(\left|e^{\lambda \phi} \mathcal{P} u\right|_{H^{-1}(Q)}^{2}+\lambda^{2} \int_{0}^{T} \int_{\omega} u^{2} e^{2 \lambda \phi} d x d t\right)
$$

where $\phi$ is the same as in Theorem 5.1.

Proof. The proof is close to that of [18, Theorem 1.1]. However, for the reader's convenience, we give the details here.

The main idea is to apply (7.1) to some special $\eta$ with $\mathcal{P} \eta=\cdots+\lambda u e^{2 \lambda \phi}$, which yields the desired term $\lambda \int_{Q} u^{2} e^{2 \lambda \phi} d x d t$ and reduces the estimate to that for $|\eta|_{H_{0}^{1}(Q)}$. We shall employ Proposition 6.1 to provide the desired $\eta$. The proof is divided into several steps. define

Step 1. First, recall the functions $\left\{\left(\hat{z}_{m}^{i}, \hat{r}_{1 m}^{i}, \hat{r}_{2 m}^{i}, \hat{r}_{m}^{i}\right)\right\}_{i=0}^{m}$ in Proposition 6.1. We

$$
\begin{aligned}
\tilde{z}^{m}(t, x) & =\frac{1}{h} \sum_{i=0}^{m-1}\left[(t-i h) \hat{z}_{m}^{i+1}(x)-(t-(i+1) h) \hat{z}_{m}^{i}(x)\right] \chi_{(i h,(i+1) h]}(t), \\
\tilde{r}_{1}^{m}(t, x) & =\hat{r}_{1 m}^{0}(x) \chi_{\{0\}}(t) \\
& +\frac{1}{h} \sum_{i=0}^{m-1}\left[(t-i h) \hat{r}_{1 m}^{i+1}(x)-(t-(i+1) h) \hat{r}_{1 m}^{i}(x)\right] \chi_{(i h,(i+1) h]}(t), \\
\tilde{r}_{2}^{m}(t, x)= & \frac{1}{h} \sum_{i=0}^{m-1}\left[(t-i h) \hat{r}_{2 m}^{i+1}(x)-(t-(i+1) h) \hat{r}_{2 m}^{i}(x)\right] \chi_{(i h,(i+1) h]}(t), \\
\tilde{r}^{m}(t, x)= & \frac{1}{h} \sum_{i=0}^{m-1}\left[(t-i h) \hat{r}_{m}^{i+1}(x)-(t-(i+1) h) \hat{r}_{m}^{i}(x)\right] \chi_{(i h,(i+1) h]}(t) .
\end{aligned}
$$

By (6.10)-(6.11), one can find a subsequence of $\left(\tilde{z}^{m}, \tilde{r}_{1}^{m}, \tilde{r}_{2}^{m}, \tilde{r}^{m}\right)$, which converges weakly to some $\left(\tilde{z}, \tilde{r}_{1}, \tilde{r}_{2}, \tilde{r}\right) \in\left(H^{1}\left(0, T ; L^{2}(\Omega)\right)\right)^{4}$, as $m \rightarrow \infty$.

For any constant $K>1$, put

$$
\tilde{p} \triangleq K \tilde{r}
$$

In what follows, we shall choose $K$ to be sufficiently large (see (7.19)). By (6.3), (6.8)-(6.11), and noting Lemma 3.1, we see that

$$
\tilde{z}, \tilde{p} \in C\left([0, T] ; H_{0}^{1}(\Omega)\right) \cap C^{1}\left([0, T] ; L^{2}(\Omega)\right)
$$

Copyright (c) by SIAM. Unauthorized reproduction of this article is prohibited. 
and

$$
\begin{cases}\mathcal{P} \tilde{z}=\tilde{r}_{1, t}+\tilde{r}_{2}+\lambda u e^{2 \lambda \phi}+\tilde{r} & \text { in } Q, \\ \mathcal{P} \tilde{p}+\tilde{z} e^{-2 \lambda \phi}=0 & \text { in } Q, \\ \tilde{p}=\tilde{z}=0 & \text { on } \Sigma, \\ \tilde{p}(0)=\tilde{p}(T)=\tilde{z}(0)=\tilde{z}(T)=0 & \text { in } \Omega, \\ \tilde{p}_{t}+\varrho \frac{\tilde{r}_{1}}{\lambda^{2}} e^{-2 \lambda \phi}=0 & \text { in } Q, \\ \tilde{p}-\varrho \frac{\tilde{r}_{2}}{\lambda^{4}} e^{-2 \lambda \phi}=0 & \text { in } Q .\end{cases}
$$

Step 2. Applying Theorem 5.1 to $\tilde{p}$ in (7.3), one gets

$$
\begin{aligned}
& \lambda \int_{Q}\left(\lambda^{2} \tilde{p}^{2}+\tilde{p}_{t}^{2}+|\nabla \tilde{p}|^{2}\right) e^{2 \lambda \phi} d x d t \\
& \leq C\left[\int_{Q} \tilde{z}^{2} e^{-2 \lambda \phi} d x d t+\lambda^{2} \int_{0}^{T} \int_{\omega}\left(\lambda^{2} \tilde{p}^{2}+\tilde{p}_{t}^{2}\right) e^{2 \lambda \phi} d x d t\right] \\
& \leq C\left[\int_{Q} \tilde{z}^{2} e^{-2 \lambda \phi} d x d t+\int_{0}^{T} \int_{\omega}\left(\frac{\tilde{r}_{1}^{2}}{\lambda^{2}}+\frac{\tilde{r}_{2}^{2}}{\lambda^{4}}\right) e^{-2 \lambda \phi} d x d t\right] .
\end{aligned}
$$

Here and henceforth, $C$ is a constant, independent of $K$ and $\lambda$.

By (7.3) again, one finds that $\tilde{p}_{t}$ satisfies

$$
\begin{cases}\mathcal{P} \tilde{p}_{t}+\left(\tilde{z} e^{-2 \lambda \phi}\right)_{t}=0 & \text { in } Q, \\ \tilde{p}_{t}=0 & \text { on } \Sigma \\ \tilde{p}_{t t}+\frac{\varrho}{\lambda}\left(\frac{\tilde{r}_{1, t}}{\lambda}-2 \phi_{t} \tilde{r}_{1}\right) e^{-2 \lambda \phi}=0 & \text { in } Q \\ \tilde{p}_{t}-\frac{\varrho}{\lambda^{2}}\left(\frac{\tilde{r}_{2, t}}{\lambda^{2}}-\frac{2}{\lambda} \phi_{t} \tilde{r}_{2}\right) e^{-2 \lambda \phi}=0 & \text { in } Q .\end{cases}
$$

Applying Theorem 5.1 to $\tilde{p}_{t}$ and noting (7.5), we obtain

$$
\begin{aligned}
& \lambda \int_{Q}\left(\lambda^{2} \tilde{p}_{t}^{2}+\tilde{p}_{t t}^{2}+\left|\nabla \tilde{p}_{t}\right|^{2}\right) e^{2 \lambda \phi} d x d t \\
& \leq C\left[\left|e^{\lambda \phi}\left(e^{-2 \lambda \phi} \tilde{z}\right)_{t}\right|_{L^{2}(Q)}^{2}+\lambda^{2} \int_{0}^{T} \int_{\omega}\left(\lambda^{2} \tilde{p}_{t}^{2}+\tilde{p}_{t t}^{2}\right) e^{2 \lambda \phi} d x d t\right] \\
& \leq C\left[\int_{Q}\left(\tilde{z}_{t}^{2}+\lambda^{2} \tilde{z}^{2}\right) e^{-2 \lambda \phi} d x d t+\int_{0}^{T} \int_{\omega}\left(\frac{\tilde{r}_{1, t}^{2}}{\lambda^{2}}+\frac{\tilde{r}_{2, t}^{2}}{\lambda^{4}}+\tilde{r}_{1}^{2}+\frac{\tilde{r}_{2}^{2}}{\lambda^{2}}\right) e^{-2 \lambda \phi} d x d t\right] .
\end{aligned}
$$

Step 3. From (7.3), and noting that

$$
-\int_{Q}\left(\tilde{r}_{1, t}+\tilde{r}_{2}\right) \tilde{p} d x d t=\int_{Q}\left(\tilde{r}_{1} \tilde{p}_{t}-\tilde{r}_{2} \tilde{p}\right) d x d t=-\int_{Q} \varrho\left(\frac{\tilde{r}_{1}^{2}}{\lambda^{2}}+\frac{\tilde{r}_{2}^{2}}{\lambda^{4}}\right) e^{-2 \lambda \phi} d x d t
$$

Copyright (c) by SIAM. Unauthorized reproduction of this article is prohibited. 
and recalling $\tilde{p}=K \tilde{r}$, we get

$$
\begin{aligned}
0= & \left(\mathcal{P} \tilde{z}-\tilde{r}_{1, t}-\tilde{r}_{2}-\lambda u e^{2 \lambda \phi}-\tilde{r}, \tilde{p}\right)_{L^{2}(Q)} \\
= & -\int_{Q} \tilde{z}^{2} e^{-2 \lambda \phi} d x d t-\int_{Q} \varrho\left(\frac{\tilde{r}_{1}^{2}}{\lambda^{2}}+\frac{\tilde{r}_{2}^{2}}{\lambda^{4}}\right) e^{-2 \lambda \phi} d x d t \\
& -\lambda \int_{Q} u \tilde{p} e^{2 \lambda \phi} d x d t-K \int_{Q} \tilde{r}^{2} d x d t .
\end{aligned}
$$

Hence

$$
\begin{aligned}
& \int_{Q} \tilde{z}^{2} e^{-2 \lambda \phi} d x d t+\int_{Q} \varrho\left(\frac{\tilde{r}_{1}^{2}}{\lambda^{2}}+\frac{\tilde{r}_{2}^{2}}{\lambda^{4}}\right) e^{-2 \lambda \phi} d x d t+K \int_{Q} \tilde{r}^{2} d x d t \\
& =-\lambda \int_{Q} u \tilde{p} e^{2 \lambda \phi} d x d t .
\end{aligned}
$$

Combining (7.4) and (7.9), we arrive at

$$
\begin{aligned}
& \int_{Q} \tilde{z}^{2} e^{-2 \lambda \phi} d x d t+\int_{Q} \varrho\left(\frac{\tilde{r}_{1}^{2}}{\lambda^{2}}+\frac{\tilde{r}_{2}^{2}}{\lambda^{4}}\right) e^{-2 \lambda \phi} d x d t+K \int_{Q} \tilde{r}^{2} d x d t \\
& \leq \frac{C}{\lambda} \int_{Q} u^{2} e^{2 \lambda \phi} d x d t .
\end{aligned}
$$

Step 4. Using (7.3) and (7.5) again, and noting $\tilde{p}_{t t}(0)=\tilde{p}_{t t}(T)=0$ in $\Omega$, we get

$$
\begin{aligned}
0= & \left(\mathcal{P} \tilde{z}-\tilde{r}_{1, t}-\tilde{r}_{2}-\lambda u e^{2 \lambda \phi}-\tilde{r}, \tilde{p}_{t t}\right)_{L^{2}(Q)} \\
= & -\int_{Q} \tilde{z}\left(e^{-2 \lambda \phi} \tilde{z}\right)_{t t} d x d t-\int_{Q}\left(\tilde{r}_{1, t}+\tilde{r}_{2}\right) \tilde{p}_{t t} d x d t \\
& -\lambda \int_{Q} u \tilde{p}_{t t} e^{2 \lambda \phi} d x d t-\int_{Q} \tilde{r} \tilde{r}_{t t} d x d t .
\end{aligned}
$$

Note

$$
\begin{aligned}
-\int_{Q} \tilde{z}\left(e^{-2 \lambda \phi} \tilde{z}\right)_{t t} d x d t & =\int_{Q}\left(\tilde{z}_{t}^{2} e^{-2 \lambda \phi}-\frac{\tilde{z}^{2}}{2}\left(e^{-2 \lambda \phi}\right)_{t t}\right) d x d t \\
& =\int_{Q}\left(\tilde{z}_{t}^{2}+\lambda \phi_{t t} \tilde{z}^{2}-2 \lambda^{2} \phi_{t}^{2} \tilde{z}^{2}\right) e^{-2 \lambda \phi} d x d t .
\end{aligned}
$$

Further, in view of the third and fourth equalities in (7.5), one has

$$
\begin{aligned}
& -\int_{Q}\left(\tilde{r}_{1, t}+\tilde{r}_{2}\right) \tilde{p}_{t t} d x d t=-\int_{Q}\left(\tilde{r}_{1, t} \tilde{p}_{t t}-\tilde{r}_{2, t} \tilde{p}_{t}\right) d x d t \\
& =\int_{Q} \tilde{r}_{1, t} \frac{\varrho}{\lambda}\left(\frac{\tilde{r}_{1, t}}{\lambda}-2 \phi_{t} \tilde{r}_{1}\right) e^{-2 \lambda \phi} d x d t+\int_{Q} \tilde{r}_{2, t} \frac{\varrho}{\lambda^{2}}\left(\frac{\tilde{r}_{2, t}}{\lambda^{2}}-\frac{2}{\lambda} \phi_{t} \tilde{r}_{2}\right) e^{-2 \lambda \phi} d x d t \\
& =\int_{Q} \varrho\left(\frac{\tilde{r}_{1, t}^{2}}{\lambda^{2}}+\frac{\tilde{r}_{2, t}^{2}}{\lambda^{4}}-\frac{2}{\lambda} \phi_{t} \tilde{r}_{1} \tilde{r}_{1, t}-\frac{2}{\lambda^{3}} \phi_{t} \tilde{r}_{2} \tilde{r}_{2, t}\right) e^{-2 \lambda \phi} d x d t
\end{aligned}
$$

Copyright $@$ by SIAM. Unauthorized reproduction of this article is prohibited. 
Moreover, by $\tilde{p} \triangleq K \tilde{r}$ and integration by parts, one gets

$$
-\int_{Q} \tilde{r} \tilde{p}_{t t} d x d t=K \int_{Q} \tilde{r}_{t}^{2} d x d t
$$

Combining (7.11)-(7.14), we end up with

$$
\begin{aligned}
& \int_{Q} \varrho\left(\frac{\tilde{r}_{1, t}^{2}}{\lambda^{2}}+\frac{\tilde{r}_{2, t}^{2}}{\lambda^{4}}-\frac{2}{\lambda} \phi_{t} \tilde{r}_{1} \tilde{r}_{1, t}-\frac{2}{\lambda^{3}} \phi_{t} \tilde{r}_{2} \tilde{r}_{2, t}\right) e^{-2 \lambda \phi} d x d t+K \int_{Q} \tilde{r}_{t}^{2} d x d t \\
& +\int_{Q}\left(\tilde{z}_{t}^{2}+\lambda \phi_{t t} \tilde{z}^{2}-2 \lambda^{2} \phi_{t}^{2} \tilde{z}^{2}\right) e^{-2 \lambda \phi} d x d t=\lambda \int_{Q} u \tilde{p}_{t t} e^{2 \lambda \phi} d x d t .
\end{aligned}
$$

Now, by $(7.15)+C \lambda^{2} \cdot(7.10)$ (with a sufficiently large $C>0$ ), using the CauchySchwarz inequality and noting (7.6), we obtain

$$
\begin{aligned}
& \int_{Q}\left(\tilde{z}_{t}^{2}+\lambda^{2} \tilde{z}^{2}\right) e^{-2 \lambda \phi} d x d t+\int_{Q} \varrho\left(\frac{\tilde{r}_{1, t}^{2}}{\lambda^{2}}+\frac{\tilde{r}_{2, t}^{2}}{\lambda^{4}}+\tilde{r}_{1}^{2}+\frac{\tilde{r}_{2}^{2}}{\lambda^{2}}\right) e^{-2 \lambda \phi} d x d t \\
& \leq C \lambda \int_{Q} u^{2} e^{2 \lambda \phi} d x d t .
\end{aligned}
$$

Step 5. By (7.3), we have

$$
\begin{aligned}
& \left(\tilde{r}_{1, t}+\tilde{r}_{2}+\lambda u e^{2 \lambda \phi}+\tilde{r}, \tilde{z} e^{-2 \lambda \phi}\right)_{L^{2}(Q)}=\left(\mathcal{P} \tilde{z}, \tilde{z} e^{-2 \lambda \phi}\right)_{L^{2}(Q)} \\
& =-\int_{Q} \tilde{z}_{t}\left(\tilde{z} e^{-2 \lambda \phi}\right)_{t} d x d t+\sum_{i, j} \int_{Q} a^{i j} \tilde{z}_{i}\left(\tilde{z} e^{-2 \lambda \phi}\right)_{j} d x d t \\
& =-\int_{Q}\left(\tilde{z}_{t}^{2}+\lambda \phi_{t t} \tilde{z}^{2}-2 \lambda^{2} \phi_{t}^{2} \tilde{z}^{2}\right) e^{-2 \lambda \phi} d x d t+\sum_{i, j} \int_{Q} a^{i j} \tilde{z}_{i} \tilde{z}_{j} e^{-2 \lambda \phi} d x d t \\
& \quad-2 \lambda \sum_{i, j} \int_{Q} a^{i j} \tilde{z}_{i} \tilde{z} \phi_{j} e^{-2 \lambda \phi} d x d t .
\end{aligned}
$$

This, combined with (1.2), yields (recall $\lambda \geq \lambda_{0}>1$ )

$$
\begin{aligned}
& \int_{Q}|\nabla \tilde{z}|^{2} e^{-2 \lambda \phi} d x d t \\
& \leq C \int_{Q}\left[\left|\tilde{r}_{1, t}+\tilde{r}_{2}+\tilde{r}\right||\tilde{z}| e^{-2 \lambda \phi}+\lambda|u \tilde{z}|+\left(\tilde{z}_{t}^{2}+\lambda^{2} \tilde{z}^{2}\right) e^{-2 \lambda \phi}\right] d x d t \\
& \leq C \int_{Q}\left[u^{2} e^{2 \lambda \phi}+\left(\frac{\tilde{r}_{1, t}^{2}}{\lambda^{2}}+\frac{\tilde{r}_{2}^{2}}{\lambda^{2}}+\tilde{r}^{2}+\tilde{z}_{t}^{2}+\lambda^{2} \tilde{z}^{2}\right) e^{-2 \lambda \phi}\right] d x d t .
\end{aligned}
$$

Combining (7.10), (7.16), and (7.18); choosing the constant $K$ in (7.10) so that

$$
K \geq C e^{2 \lambda \max _{(t, x) \in Q}|\phi|}
$$

(to absorb the term $C \int_{Q} \tilde{r}^{2} e^{-2 \lambda \phi} d x d t$ in the right-hand side of (7.18)); and noting that $\varrho(x) \geq 1$ in $\Omega$, we deduce that

$$
\begin{aligned}
& \int_{Q}\left(|\nabla \tilde{z}|^{2}+\tilde{z}_{t}^{2}+\lambda^{2} \tilde{z}^{2}\right) e^{-2 \lambda \phi} d x d t+\int_{Q} \varrho\left(\frac{\tilde{r}_{1, t}^{2}}{\lambda^{2}}+\frac{\tilde{r}_{2, t}^{2}}{\lambda^{4}}+\tilde{r}_{1}^{2}+\frac{\tilde{r}_{2}^{2}}{\lambda^{2}}\right) e^{-2 \lambda \phi} d x d t \\
& \leq C \lambda \int_{Q} u^{2} e^{2 \lambda \phi} d x d t .
\end{aligned}
$$

Copyright $@$ by SIAM. Unauthorized reproduction of this article is prohibited. 
Step 6. Recall that $\left(\tilde{z}, \tilde{r}_{1}, \tilde{r}_{2}, \tilde{r}\right)$ depend on $K$. We now fix $\lambda$ and let $K \rightarrow \infty$. By (7.10) and (7.20), we conclude that there exists a subsequence of $\left(\tilde{z}, \tilde{r}_{1}, \tilde{r}_{2}, \tilde{r}\right)$ which converges weakly to some $\left(\check{z}, \check{r}_{1}, \check{r}_{2}, 0\right)$ in $H_{0}^{1}(Q) \times\left(H^{1}\left(0, T ; L^{2}(\Omega)\right)\right)^{2} \times L^{2}(Q)$, with supp $\check{r}_{i} \subset \overline{(0, T) \times \omega}(i=1,2)$ since $\varrho(x) \equiv \varrho^{K}(x) \rightarrow \infty$ for any $x \notin \omega$, as $K \rightarrow \infty$. By (7.3), we deduce that $\left(\check{z}, \check{r}_{1}, \check{r}_{2}\right)$ satisfies

$$
\begin{cases}\mathcal{P} \check{z}=\check{r}_{1, t}+\check{r}_{2}+\lambda u e^{2 \lambda \phi} & \text { in } Q, \\ \check{z}=0 & \text { on } \partial Q .\end{cases}
$$

Using (7.20) again, we find

$$
\left|\check{z} e^{-\lambda \phi}\right|_{H_{0}^{1}(Q)}^{2}+\frac{1}{\lambda^{2}} \int_{0}^{T} \int_{\omega}\left(\check{r}_{1, t}^{2}+\check{r}_{2}^{2}\right) e^{-2 \lambda \phi} d x d t \leq C \lambda \int_{Q} u^{2} e^{2 \lambda \phi} d x d t .
$$

Now, by (7.1) with $\eta$ replaced by the above $\check{z}$, one gets

$$
\left(u, \check{r}_{1, t}+\check{r}_{2}+\lambda u e^{2 \lambda \phi}\right)_{L^{2}(Q)}=\langle\mathcal{P} u, \check{z}\rangle_{H^{-1}(Q), H_{0}^{1}(Q)} .
$$

Hence, noting supp $\check{r}_{i} \subset \overline{(0, T) \times \omega}(i=1,2)$, we conclude that for any $\varepsilon>0$, it holds that

$$
\begin{aligned}
& \lambda \int_{Q} u^{2} e^{2 \lambda \phi} d x d t=\langle\mathcal{P} u, \check{z}\rangle_{H^{-1}(Q), H_{0}^{1}(Q)}-\left(u, \check{r}_{1, t}+\check{r}_{2}\right)_{L^{2}((0, T) \times \omega)} \\
& \leq C\left\{\frac{1}{\varepsilon}\left[\left|e^{\lambda \phi} \mathcal{P} u\right|_{H^{-1}(Q)}^{2}+\lambda^{2} \int_{0}^{T} \int_{\omega} u^{2} e^{2 \lambda \phi} d x d t\right]\right. \\
& \left.\quad+\varepsilon\left[\left|\check{z} e^{-\lambda \phi}\right|_{H_{0}^{1}(Q)}^{2}+\frac{1}{\lambda^{2}} \int_{0}^{T} \int_{\omega}\left(\check{r}_{1, t}^{2}+\check{r}_{2}^{2}\right) e^{-2 \lambda \phi} d x d t\right]\right\} .
\end{aligned}
$$

Finally, choosing $\varepsilon$ in (7.23) sufficiently small and noting (7.22), we arrive at the desired estimate (7.2). This completes the proof of Theorem 7.1.

8. Proof of Theorem 2.3. The main idea is to use the Carleman estimate in Theorem 7.1. Note, however, that our $w$ satisfying (1.7) does not necessarily vanish at $t=0, T$. Therefore we need to introduce a suitable cutoff function. To this end, set

$$
\left\{\begin{array}{l}
T_{i} \triangleq T / 2-\varepsilon_{i} T, \quad T_{i}^{\prime} \triangleq T / 2+\varepsilon_{i} T \\
R_{0} \triangleq \min _{x \in \bar{\Omega}} \sqrt{d(x)}(>0)
\end{array}\right.
$$

where $i=0,1 ; 0<\varepsilon_{0}<\varepsilon_{1}<1 / 2$ will be given below.

From (5.2) and (4.13), it is easy to see that

$$
\phi(0, x)=\phi(T, x)<R_{1}^{2}-c T^{2} / 4<0 \quad \forall x \in \Omega .
$$

Therefore there exists an $\varepsilon_{1} \in(0,1 / 2)$ close to $1 / 2$ such that

$$
\phi(t, x) \leq R_{1}^{2} / 2-c T^{2} / 8<0 \quad \forall(t, x) \in\left(\left(0, T_{1}\right) \bigcup\left(T_{1}^{\prime}, T\right)\right) \times \Omega
$$

Copyright (c) by SIAM. Unauthorized reproduction of this article is prohibited. 
with $T_{1}$ and $T_{1}^{\prime}$ given by (8.1). Further, by (4.13), we see that

$$
\phi(T / 2, x)=d(x) \geq R_{0}^{2} \quad \forall x \in \Omega
$$

Hence, one can find an $\varepsilon_{0} \in(0,1 / 2)$, close to 0 , such that

$$
\phi(t, x) \geq R_{0}^{2} / 2 \quad \forall(t, x) \in\left(T_{0}, T_{0}^{\prime}\right) \times \Omega,
$$

with $T_{0}$ and $T_{0}^{\prime}$ given by (8.1). We now choose a nonnegative function $\xi \in C_{0}^{\infty}(0, T)$ so that

$$
\xi(t) \equiv 1 \quad \text { in }\left(T_{1}, T_{1}^{\prime}\right)
$$

Clearly, $\xi w$ vanishes at $t=0, T$. Hence, by Theorem 7.1, for any $\lambda \geq \lambda_{0}$, we have

$$
\lambda \int_{Q}(\xi w)^{2} e^{2 \lambda \phi} d x d t \leq C\left(\left|e^{\lambda \phi} \mathcal{P}(\xi w)\right|_{H^{-1}(Q)}^{2}+\lambda^{2} \int_{0}^{T} \int_{\omega} w^{2} e^{2 \lambda \phi} d x d t\right)
$$

By (1.7), we have

$$
\begin{aligned}
& \left|e^{\lambda \phi} \mathcal{P}(\xi w)\right|_{H^{-1}(Q)}=\mid e^{\lambda \phi}\left(\xi \mathcal{P} w+2 \xi_{t} w_{t}+\left.w \xi_{t t}\right|_{H^{-1}(Q)}\right. \\
& =\left|e^{\lambda \phi}\left(\xi q w+2 \xi_{t} w_{t}+w \xi_{t t}\right)\right|_{H^{-1}(Q)} \\
& =\sup _{|f|_{H_{0}^{1}(Q)}=1}\left\langle e^{\lambda \phi}\left(\xi q w+2 \xi_{t} w_{t}+w \xi_{t t}\right), f\right\rangle_{H^{-1}(Q), H_{0}^{1}(Q)} \\
& \leq \sup _{|f|_{H_{0}^{1}(Q)}=1} \int_{Q} e^{\lambda \phi} \xi q w f d x d t \\
& \quad+\sup _{|f|_{H_{0}^{1}(Q)}=1}\left\langle e^{\lambda \phi}\left(2 \xi_{t} w_{t}+w \xi_{t t}\right), f\right\rangle_{H^{-1}(Q), H_{0}^{1}(Q)} .
\end{aligned}
$$

Using the Sobolev embedding theorem and the Hölder inequality, and recalling $r \triangleq|q|_{L^{\infty}\left(0, T ; L^{n}(\Omega)\right)}$, we get

$$
\sup _{|f|_{H_{0}^{1}(Q)}=1} \int_{Q} e^{\lambda \phi} \xi q w f d x d t \leq C r\left|e^{\lambda \phi} w\right|_{L^{2}(Q)}
$$

On the other hand, by (8.3) and (8.5), we have

$$
\begin{aligned}
& \sup _{|f|_{H_{0}^{1}(Q)}=1}\left\langle e^{\lambda \phi}\left(2 \xi_{t} w_{t}+w \xi_{t t}\right), f\right\rangle_{H^{-1}(Q), H_{0}^{1}(Q)} \\
& =\sup _{|f|_{H_{0}^{1}(Q)}=1} \int_{Q} e^{\lambda \phi} w\left(-\xi_{t t} f-2 \xi_{t} f_{t}-2 \lambda \phi_{t} \xi_{t} f\right) d x d t \\
& \leq C e^{\left(R_{1}^{2} / 2-c T^{2} / 8\right) \lambda}(1+\lambda)\left(|w|_{L^{2}\left(\left(0, T_{1}\right) \times \Omega\right)}+|w|_{L^{2}\left(\left(T_{1}^{\prime}, T\right) \times \Omega\right)}\right) .
\end{aligned}
$$

Copyright $@$ by SIAM. Unauthorized reproduction of this article is prohibited. 
Further, by (8.3) and (8.5), we have

$$
\begin{aligned}
& \int_{Q}(\xi w)^{2} e^{2 \lambda \phi} d x d t=\int_{Q} w^{2} e^{2 \lambda \phi} d x d t-\int_{Q}\left(1-\xi^{2}\right) w^{2} e^{2 \lambda \phi} d x d t \\
& =\int_{Q} w^{2} e^{2 \lambda \phi} d x d t-\int_{0}^{T_{1}} \int_{\Omega}\left(1-\xi^{2}\right) w^{2} e^{2 \lambda \phi} d x d t \\
& \quad-\int_{T_{1}^{\prime}}^{T} \int_{\Omega}\left(1-\xi^{2}\right) w^{2} e^{2 \lambda \phi} d x d t \\
& \geq \int_{Q} w^{2} e^{2 \lambda \phi} d x d t-C e^{\left(R_{1}^{2}-c T^{2} / 4\right) \lambda}\left(|w|_{L^{2}\left(\left(0, T_{1}\right) \times \Omega\right)}^{2}+|w|_{L^{2}\left(\left(T_{1}^{\prime}, T\right) \times \Omega\right)}^{2} .\right.
\end{aligned}
$$

Combining (8.6)-(8.10), we arrive at

$$
\begin{aligned}
& \lambda \int_{Q} w^{2} e^{2 \lambda \phi} d x d t \\
& \leq C_{1}\left[r^{2} \int_{Q} w^{2} e^{2 \lambda \phi} d x d t+\lambda^{2} \int_{0}^{T} \int_{\omega} w^{2} e^{2 \lambda \phi} d x d t\right. \\
& \left.\quad+e^{\left(R_{1}^{2}-c T^{2} / 4\right) \lambda}\left(1+\lambda^{2}\right)\left(|w|_{L^{2}\left(\left(0, T_{1}\right) \times \Omega\right)}^{2}+|w|_{L^{2}\left(\left(T_{1}^{\prime}, T\right) \times \Omega\right)}^{2}\right)\right],
\end{aligned}
$$

for a constant $C_{1}>0$, independent of $\lambda$ and $r$. Since $R_{1}^{2}-c T^{2} / 4<0$, one may find a $\lambda_{1} \geq \lambda_{0}$ such that $e^{\left(R_{1}^{2}-c T^{2} / 4\right) \lambda}\left(1+\lambda^{2}\right)<1$ for all $\lambda \geq \lambda_{1}$. Now, taking

$$
\lambda \geq 2 C_{1}\left(\lambda_{1}+r^{2}\right),
$$

it follows from (8.11) that

$$
\begin{aligned}
& \lambda \int_{Q} w^{2} e^{2 \lambda \phi} d x d t \\
& \leq C\left(\lambda^{2} \int_{0}^{T} \int_{\omega} w^{2} e^{2 \lambda \phi} d x d t+|w|_{L^{2}\left(\left(0, T_{1}\right) \times \Omega\right)}^{2}+|w|_{L^{2}\left(\left(T_{1}^{\prime}, T\right) \times \Omega\right)}^{2}\right) .
\end{aligned}
$$

From (8.4), we see that

$$
\int_{Q} w^{2} e^{2 \lambda \phi} d x d t \geq e^{R_{0}^{2} \lambda} \int_{T_{0}}^{T_{0}^{\prime}} \int_{\Omega} w^{2} d x d t
$$

For any $S_{0} \in\left(T_{0}, T / 2\right)$ and $S_{0}^{\prime} \in\left(T / 2, T_{0}^{\prime}\right)$, by Lemma 3.4, we obtain (recall (3.4) for $E(t))$

$$
\int_{S_{0}}^{S_{0}^{\prime}} E(t) d t \leq C(1+r) \int_{T_{0}}^{T_{0}^{\prime}} \int_{\Omega} w^{2} d x d t
$$

On the other hand, by Lemma 3.3, we have

$$
|w|_{L^{2}\left(\left(0, T_{1}\right) \times \Omega\right)}^{2}+|w|_{L^{2}\left(\left(T_{1}^{\prime}, T\right) \times \Omega\right)}^{2} \leq C E(0) e^{C r}
$$

Copyright $@$ by SIAM. Unauthorized reproduction of this article is prohibited. 
and

$$
\int_{S_{0}}^{S_{0}^{\prime}} E(t) d t \geq C E(0) e^{C r}
$$

Combining (8.13)-(8.17), we end up with

$$
\left(C_{2} \lambda e^{R_{0}^{2} \lambda+C_{2} r}-C_{3}(1+r) e^{C_{3} r}\right) E(0) \leq C \lambda^{2}(1+r) e^{C \lambda} \int_{0}^{T} \int_{\omega} w^{2} d x d t,
$$

for two constants $C_{2}>0$ and $C_{3}>0$, independent of $\lambda$ and $r$. We now choose $\lambda$ so that

$$
C_{2} \lambda \geq C_{3}(1+r), \quad R_{0}^{2} \lambda+C_{2} r \geq C_{3} r .
$$

Then, from (8.18), we obtain

$$
E(0) \leq \mathcal{C}(q)|w|_{L^{2}((0, T) \times \omega)}^{2} .
$$

Finally, noting (8.12) and (8.19), we conclude (2.12). This completes the proof of Theorem 2.3.

9. Proof of Theorem 2.2. The proof is very close to that of [26, Theorem 3.1] and [38, Theorem 2.1]. However, for the reader's convenience, we give some details here.

Define a function $h(\cdot) \in C(\mathbb{R})$ by

$$
h(s) \triangleq \begin{cases}{[f(s)-f(0)] / s} & \text { if } s \neq 0 \\ f^{\prime}(0) & \text { if } s=0 .\end{cases}
$$

Let the initial and final data $\left(y_{0}, y_{1}\right),\left(z_{0}, z_{1}\right) \in H_{0}^{1}(\Omega) \times L^{2}(\Omega)$ be given. For any given $z(\cdot) \in L^{\infty}\left(0, T ; L^{2}(\Omega)\right)$, we look for a control $\gamma=\gamma(z(\cdot)) \in L^{2}((0, T) \times \omega)$ such that the solution $y=y(\cdot ; z(\cdot))$ of

$$
\begin{cases}\mathcal{P} y=h(z(\cdot)) y+f(0)+\chi_{\omega}(x) \gamma(t, x) & \text { in } Q, \\ y=0 & \text { on } \Sigma, \\ y(0)=y_{0}, \quad y_{t}(0)=y_{1} & \text { in } \Omega\end{cases}
$$

satisfies

$$
y(T)=z_{0}, \quad y_{t}(T)=z_{1} \quad \text { in } \Omega .
$$

For this purpose, we use the classical duality argument [29, 28, 39]. First, we solve

$$
\begin{cases}\mathcal{P} v=h(z(\cdot)) v+f(0) & \text { in } Q, \\ v=0 & \text { on } \Sigma, \\ v(T)=z_{0}, \quad v_{t}(T)=z_{1} & \text { in } \Omega,\end{cases}
$$

which admits a unique weak solution $v=v(\cdot ; z(\cdot)) \in C\left([0, T] ; H_{0}^{1}(\Omega)\right) \cap C^{1}\left([0, T] ; L^{2}(\Omega)\right)$. Next, put $X \triangleq L^{2}(\Omega) \times H^{-1}(\Omega)$. For any $\left(w_{0}, w_{1}\right) \in X$, we solve

$$
\begin{cases}\mathcal{P} w=h(z(\cdot)) w & \text { in } Q, \\ w=0 & \text { on } \Sigma, \\ w(0)=w_{0}, \quad w_{t}(0)=w_{1} & \text { in } \Omega\end{cases}
$$

Copyright $@$ by SIAM. Unauthorized reproduction of this article is prohibited. 
and

$$
\begin{cases}\mathcal{P} \eta=h(z(\cdot)) \eta+\chi_{\omega}(x) w(t, x) & \text { in } Q, \\ \eta=0 & \text { on } \Sigma, \\ \eta(T)=\eta_{t}(T)=0 & \text { in } \Omega .\end{cases}
$$

Now, we define a linear and continuous operator $\Lambda: X \rightarrow X^{\prime}$, the dual space of $X$, by

$$
\Lambda\left(w_{0}, w_{1}\right) \triangleq\left(-\eta_{t}(0), \eta(0)\right)
$$

where $\eta \in C\left([0, T] ; H_{0}^{1}(\Omega)\right) \cap C^{1}\left([0, T] ; L^{2}(\Omega)\right)$ is the weak solution of (9.6).

Let us show the existence of some $\left(w_{0}, w_{1}\right) \in X$ such that

$$
\Lambda\left(w_{0}, w_{1}\right)=\left(-y_{1}+v_{t}(0), y_{0}-v(0)\right) .
$$

For this purpose, we observe that, by multiplying the first equation in (9.6) by $w$; integrating it in $Q$; using integration by parts; and noting $(9.5), \eta(T)=\eta_{t}(T)=0$ in $\Omega$, and (9.7), it follows that

$$
\left\langle\Lambda\left(w_{0}, w_{1}\right),\left(w_{0}, w_{1}\right)\right\rangle_{X^{\prime}, X}=\int_{0}^{T} \int_{\omega} w^{2} d x d t .
$$

However, by Theorem 2.3 and (9.9), we have

$$
\left\langle\Lambda\left(w_{0}, w_{1}\right),\left(w_{0}, w_{1}\right)\right\rangle_{X^{\prime}, X} \geq \frac{1}{\mathcal{C}(h(z(\cdot)))}\left|\left(w_{0}, w_{1}\right)\right|_{X}^{2} \quad \forall\left(w_{0}, w_{1}\right) \in X,
$$

where $\mathcal{C}(\cdot)$ is the constant given in (2.12). By the Lax-Milgram theorem, (9.8) admits a unique solution $\left(w_{0}, w_{1}\right) \in X$. It is easy to check that

$$
\gamma=w
$$

is the desired control such that the weak solution $y \equiv v+\eta$ of (9.2) satisfies (9.3).

Further, proceeding as in the proof of [38, Theorem 2.1], by (9.10) we end up with

$$
\begin{aligned}
& |w|_{C\left([0, T] ; L^{2}(\Omega)\right)} \\
& \leq \mathcal{C}(h(z(\cdot)))\left(|f(0)|+\left|\left(y_{0}, y_{1}\right)\right|_{H_{0}^{1}(\Omega) \times L^{2}(\Omega)}+\left|\left(z_{0}, z_{1}\right)\right|_{H_{0}^{1}(\Omega) \times L^{2}(\Omega)}\right) .
\end{aligned}
$$

Next, similarly to the proof of [26, Theorem 3.1] by applying the classical energy method to (9.2), noting (9.11)-(9.12), and recalling assumption (1.4), one concludes that there is a constant $C>0$ such that, for any $\varepsilon \in(0,4]$, it holds that

$$
\begin{aligned}
& |y|_{C\left([0, T] ; H_{0}^{1}(\Omega)\right) \cap C^{1}\left([0, T] ; L^{2}(\Omega)\right)} \\
& \leq C\left[|f(0)|+\left|\left(y_{0}, y_{1}\right)\right|_{H_{0}^{1}(\Omega) \times L^{2}(\Omega)}\right. \\
& \left.\quad+\left|\left(z_{0}, z_{1}\right)\right|_{H_{0}^{1}(\Omega) \times L^{2}(\Omega)}\right]\left(1+|z|_{L^{\infty}\left(0, T ; L^{2}(\Omega)\right)}^{4 /(1+\varepsilon)}\right) .
\end{aligned}
$$

Consequently if we take $\varepsilon=4$ in (9.13), the desired exact controllability result follows from the fixed point technique. This completes the proof of Theorem 2.2. 
10. Appendix A. Proof of Proposition 2.1. Consider first the case when $A=\operatorname{diag}\left[a^{1}, \ldots, a^{n}\right]$ with $a^{i} \in C^{1}(\bar{\Omega}) \quad(i=1, \ldots, n)$. In this case, the matrix $\mathcal{A}$ (defined in (2.3)) reads

$$
\mathcal{A}=\left(a^{i} a^{j} d_{i j}+\frac{a^{i} a_{i}^{j} d_{j}+a^{j} a_{j}^{i} d_{i}}{2}\right)_{1 \leq i, j \leq n}-\frac{1}{2} \operatorname{diag}\left[\sum_{k} a^{k} a_{k}^{1} d_{k}, \ldots, \sum_{k} a^{k} a_{k}^{n} d_{k}\right] .
$$

In particular, when $n=2$ and $a^{1}$ is independent of $x_{2}$ (hence $a_{2}^{1} \equiv 0$ ), the above $\mathcal{A}$ is specialized as

$$
\begin{aligned}
\mathcal{A} & =\left(\begin{array}{ll}
\left(a^{1}\right)^{2} d_{11}+\frac{a^{1} a_{1}^{1} d_{1}-a^{2} a_{2}^{1} d_{2}}{2} & a^{1} a^{2} d_{12}+\frac{a^{1} a_{1}^{2} d_{2}+a^{2} a_{2}^{1} d_{1}}{2} \\
a^{1} a^{2} d_{12}+\frac{a^{1} a_{1}^{2} d_{2}+a^{2} a_{2}^{1} d_{1}}{2} & \left(a^{2}\right)^{2} d_{22}+\frac{a^{2} a_{2}^{2} d_{2}-a^{1} a_{1}^{2} d_{1}}{2}
\end{array}\right) \\
& =\left(\begin{array}{cc}
\left(a^{1}\right)^{2} d_{11}+\frac{a^{1} a_{1}^{1} d_{1}}{2} & a^{1} a^{2} d_{12}+\frac{a^{1} a_{1}^{2} d_{2}}{2} \\
a^{1} a^{2} d_{12}+\frac{a^{1} a_{1}^{2} d_{2}}{2} & \left(a^{2}\right)^{2} d_{22}+\frac{a^{2} a_{2}^{2} d_{2}-a^{1} a_{1}^{2} d_{1}}{2}
\end{array}\right) \\
& \equiv\left(\begin{array}{ll}
\hat{a}^{11} & \hat{a}^{12} \\
\hat{a}^{12} & \hat{a}^{22}
\end{array}\right) .
\end{aligned}
$$

Put $L=2 \operatorname{diam} \Omega$. For any parameters $\tau>0$ and $\mu>0$, we now choose $d$ to be of the form

$$
d\left(x_{1}, x_{2}\right)=e^{-\tau a^{1}\left(x_{1}\right)}+e^{-\mu\left(L+x_{2}\right)} .
$$

Then,

$$
\begin{aligned}
& d_{1}=-\tau a_{1}^{1} e^{-\tau a^{1}}, \quad d_{11}=\tau\left(\tau\left|a_{1}^{1}\right|^{2}-a_{11}^{1}\right) e^{-\tau a^{1}}, \\
& d_{12}=0, \quad d_{2}=-\mu e^{-\mu\left(L+x_{2}\right)}, \quad d_{22}=\mu^{2} e^{-\mu\left(L+x_{2}\right)} .
\end{aligned}
$$

We consider only the case when there is an $x_{0} \in G$ such that $a_{1}^{1}\left(x_{1}^{0}\right)=0, a_{11}^{1}\left(x_{1}^{0}\right)<$ $0,\left|a_{1}^{1}\right| \neq 0$ in $G \backslash\left\{x_{1}^{0}\right\}$ (the case when $a_{1}^{1}\left(x_{1}\right) \neq 0$ for any $x_{1} \in G$ is easier to analyze). By (10.1), (10.2), and noting that $a^{1}$ is uniformly positive in $\bar{\Omega}$, one may choose a sufficiently large $\tau$ such that

$$
\begin{aligned}
\hat{a}^{11} & =\left(a^{1}\right)^{2} d_{11}+\frac{a^{1} a_{1}^{1} d_{1}}{2} \\
& =\tau\left[\left(\tau\left(a^{1}\right)^{2}-\frac{a^{1}}{2}\right)\left|a_{1}^{1}\right|^{2}-a_{11}^{1}\left(a^{1}\right)^{2}\right] e^{-\tau a^{1}}>0
\end{aligned}
$$

uniformly in $\bar{\Omega}$.

Further, by (10.1) and (10.2), by noting that $a_{1}^{1} a_{1}^{2} \geq 0$, and by noting that $a^{2}$ is uniformly positive in $\bar{\Omega}$, one may choose a sufficiently large $\mu$ such that

$$
\begin{aligned}
\hat{a}^{22} & =\left(a^{2}\right)^{2} d_{22}+\frac{a^{2} a_{2}^{2} d_{2}-a^{1} a_{1}^{2} d_{1}}{2} \\
& =\left[\left(a^{2}\right)^{2} \mu^{2}-\frac{a^{2} a_{2}^{2} \mu}{2}\right] e^{-\mu\left(L+x_{2}\right)}+\frac{a^{1} a_{1}^{1} a_{1}^{2} \tau}{2} e^{-\tau a^{1}}>0
\end{aligned}
$$

uniformly in $\bar{\Omega}$. 
Further, we have

$$
\hat{a}^{12}=a^{1} a^{2} d_{12}+\frac{a^{1} a_{1}^{2} d_{2}}{2}=-\frac{a^{1} a_{1}^{2} \mu}{2} e^{-\mu\left(L+x_{2}\right)} .
$$

Now, fixing the parameter $\tau$, it is easy to see that

$$
\hat{a}^{11} \hat{a}^{22}-\left(\hat{a}^{12}\right)^{2}>0 \quad \text { uniformly in } \bar{\Omega},
$$

provided that $\mu$ is large enough (because $\left(\hat{a}^{12}\right)^{2}$ is an infinitesimal of higher order, compared to $\hat{a}^{11} \hat{a}^{12}$, with respect to large $\mu$ ). By (10.3)-(10.5), we deduce that the matrix $\mathcal{A}$ in (10.1) is uniformly positive definite in $\bar{\Omega}$.

It is clear that $\min _{x \in \bar{\Omega}}|\nabla d(x)|>0$. Therefore, Condition 2.1 holds for the above constructed function $d$.

11. Appendix B. Proof of Theorem 5.1. The proof is long and we divide it into several steps.

Step 1. Applying Corollary 4.2 to our present $u$ and $d$, we conclude that for any constants $\lambda>0$ and $k \in(0,1)$, it holds that

$$
\begin{aligned}
& \theta^{2}|\mathcal{P} u|^{2}+ M_{t} \\
&+2 \sum_{j}\left\{2 \sum_{i, i^{\prime}, j^{\prime}} a^{i j} a^{i^{\prime} j^{\prime}} \ell_{i^{\prime}} v_{i} v_{j^{\prime}}-\sum_{i, i^{\prime}, j^{\prime}} a^{i j} a^{i^{\prime} j^{\prime}} \ell_{i} v_{i^{\prime}} v_{j^{\prime}}+\Psi v \sum_{i} a^{i j} v_{i}\right. \\
&\left.\quad-2 \ell_{t} v_{t} \sum_{i} a^{i j} v_{i}+\sum_{i} a^{i j} \ell_{i} v_{t}^{2}-\sum_{i} a^{i j}\left[(\Lambda+\Psi) \ell_{i}+\frac{\Psi_{i}}{2}\right] v^{2}\right\}_{j} \\
& \geq 2 \lambda(1-k) v_{t}^{2}+B v^{2} \\
&+2 \lambda \sum_{i, j}\left\{(k-1-4 c) a^{i j}+\sum_{i^{\prime}, j^{\prime}}\left[2 a^{i j^{\prime}}\left(a^{i^{\prime} j} d_{i^{\prime}}\right)_{j^{\prime}}-a_{j^{\prime}}^{i j}{ }^{i^{\prime} j^{\prime}} d_{i^{\prime}}\right]\right\} v_{i} v_{j},
\end{aligned}
$$

where

$$
\left\{\begin{aligned}
M \triangleq 2 & {\left[\ell_{t}\left(v_{t}^{2}+\sum_{i, j} a^{i j} v_{i} v_{j}\right)-2 \sum_{i, j} a^{i j} \ell_{i} v_{j} v_{t}\right.} \\
& \left.-\Psi v v_{t}+\left((\Lambda+\Psi) \ell_{t}+\frac{\Psi_{t}}{2}\right) v^{2}\right], \\
\Psi \triangleq \lambda & {\left[\sum_{i, j}\left(a^{i j} d_{i}\right)_{j}-2 c-1+k\right], \quad \ell \triangleq \lambda \phi, \quad v \triangleq \theta u, \quad \theta \triangleq e^{\ell}, } \\
\Lambda=\lambda^{2} & {\left[4 c^{2}(t-T / 2)^{2}-\sum_{i, j} a^{i j} d_{i} d_{j}\right]+\lambda(4 c+1-k), } \\
B=2 \lambda^{3}\left[(4 c+1-k) \sum_{i^{\prime}, j^{\prime}} a^{i^{\prime} j^{\prime}} d_{i^{\prime}} d_{j^{\prime}}+\sum_{i, j} a^{i j} d_{i}\left(\sum_{i^{\prime}, j^{\prime}} a^{i^{\prime} j^{\prime}} d_{i^{\prime}} d_{j^{\prime}}\right)_{j}\right. & \\
& \left.-4(8 c+1-k) c^{2}(t-T / 2)^{2}\right]+O\left(\lambda^{2}\right) .
\end{aligned}\right.
$$

Copyright $@$ by SIAM. Unauthorized reproduction of this article is prohibited. 
Next, fix a $k$ with $4 c-3<k<1$. Hence

$$
1-k>0
$$

On the other hand, by Condition 2.1 and noting (2.1) with $\mu_{0} \geq 4$, we get

$$
\begin{aligned}
& \sum_{i, j}\left\{(k-1-4 c) a^{i j}+\sum_{i^{\prime}, j^{\prime}}\left[2 a^{i j^{\prime}}\left(a^{i^{\prime} j} d_{i^{\prime}}\right)_{j^{\prime}}-a_{j^{\prime}}^{i j} a^{i^{j^{\prime} j^{\prime}}} d_{i^{\prime}}\right]\right\} v_{i} v_{j} \\
& \geq\left(k-4 c-1+\mu_{0}\right) \sum_{i, j} a^{i j} v_{i} v_{j} \\
& =\mu \sum_{i, j} a^{i j} v_{i} v_{j} \quad \forall x \in \Omega,
\end{aligned}
$$

where

$$
\mu=\mu_{0}-1+k-4 c \geq 3+k-4 c>0 .
$$

Recalling that $d$ satisfies (2.1), and noting $a^{i^{\prime} j^{\prime}}=a^{j^{\prime} i^{\prime}}$, we find

$$
\begin{aligned}
& \mu_{0} \sum_{i, j} a^{i j} d_{i} d_{j} \leq \sum_{i, j, i^{\prime}, j^{\prime}}\left[2 a^{i j^{\prime}}\left(a^{i^{\prime} j} d_{i^{\prime}}\right)_{j^{\prime}}-a_{j^{\prime}}^{i j} a^{i^{\prime} j^{\prime}} d_{i^{\prime}}\right] d_{i} d_{j} \\
& =\sum_{i, j, i^{\prime}, j^{\prime}}\left[2 a^{i j^{\prime}} a_{j^{\prime}}^{i^{\prime} j} d_{i^{\prime}} d_{i} d_{j}+2 a^{i j^{\prime}} a^{i^{\prime} j} d_{i^{\prime} j^{\prime}} d_{i} d_{j}-a_{j^{\prime}}^{i j} a^{i^{\prime} j^{\prime}} d_{i^{\prime}} d_{i} d_{j}\right] \\
& =\sum_{i, j, i^{\prime}, j^{\prime}}\left[a^{i j^{\prime}} a_{j^{\prime}}^{i^{\prime} j} d_{i^{\prime}} d_{i} d_{j}+2 a^{i j^{\prime}} a^{i^{\prime} j} d_{i^{\prime} j^{\prime}} d_{i} d_{j}\right] \\
& =\sum_{i, j, i^{\prime}, j^{\prime}}\left[a^{i j} a_{j}^{i^{\prime} j^{\prime}} d_{i^{\prime}} d_{i} d_{j^{\prime}}+2 a^{i j} a^{i^{\prime} j^{\prime}} d_{i^{\prime} j} d_{i} d_{j^{\prime}}\right] \\
& =\sum_{i, j, i^{\prime}, j^{\prime}}\left[a^{i j} a_{j}^{i^{\prime} j^{\prime}} d_{i^{\prime}} d_{i} d_{j^{\prime}}+a^{i j} a^{i^{\prime} j^{\prime}} d_{i^{\prime} j} d_{i} d_{j^{\prime}}+a^{i j} a^{j^{\prime} i^{\prime}} d_{j^{\prime} j} d_{i} d_{i^{\prime}}\right] \\
& =\sum_{i, j} a^{i j} d_{i}\left(\sum_{i^{\prime}, j^{\prime}} a^{i^{\prime} j^{\prime}} d_{i^{\prime}} d_{j^{\prime}}\right)_{j} .
\end{aligned}
$$

Hence, recalling, respectively, (2.8) and (11.2) for $R_{1}$ and $B$, by (11.6) and using the third inequality in (2.7), and noting that $A$ is positive definite and $4 c+1-k+\mu_{0}>$ $8 c+1-k$, we arrive at

$$
\begin{aligned}
B & \geq 2 \lambda^{3}\left\{\left(4 c+1-k+\mu_{0}\right) \sum_{i, j} a^{i j} d_{i} d_{j}-4(8 c+1-k) c^{2}(t-T / 2)^{2}\right\}+O\left(\lambda^{2}\right) \\
& \geq 2 \lambda^{3}(8 c+1-k)\left[\sum_{i, j} a^{i j} d_{i} d_{j}-4 c^{2}(t-T / 2)^{2}\right]+O\left(\lambda^{2}\right) \\
& \geq 16 c\left(4 R_{1}^{2}-c^{2} T^{2}\right) \lambda^{3}+O\left(\lambda^{2}\right) .
\end{aligned}
$$

Copyright (c) by SIAM. Unauthorized reproduction of this article is prohibited. 
Note that, by (5.2), the constant $16 c\left(4 R_{1}^{2}-c^{2} T^{2}\right)$ in (11.7) is positive. Hence, by choosing a suitable $\lambda_{0}>1$, for any $\lambda \geq \lambda_{0}$, we have

$$
B \geq 8 c\left(4 R_{1}^{2}-c^{2} T^{2}\right) \lambda^{3} .
$$

Step 2. Integrating (11.1) on $Q$, using integration by parts, recalling (11.3)-(11.5) and (11.8), and noting that $v_{i}=\frac{\partial v}{\partial \nu} \nu_{i}$ on $\Sigma$ (which follows from $\left.v\right|_{\Sigma}=0$ ), we arrive at (recall (11.2) for $M=M(t, x)$ )

$$
\begin{aligned}
& \lambda \int_{Q}\left(\lambda^{2} v^{2}+v_{t}^{2}+\sum_{i, j} a^{i j} v_{i} v_{j}\right) d x d t \\
& \leq C\left[\int_{Q} \theta^{2}|\mathcal{P} u|^{2} d x d t+\int_{\Omega} M(T, x) d x-\int_{\Omega} M(0, x) d x\right. \\
& \left.\quad+\lambda \int_{\Sigma}\left(\sum_{i, j} a^{i j} \nu_{i} \nu_{j}\right)\left(\sum_{i^{\prime}, j^{\prime}} a^{i^{\prime} j^{\prime}} d_{i^{\prime}} \nu_{j^{\prime}}\right)\left|\frac{\partial v}{\partial \nu}\right|^{2} d x d t\right] \quad \forall \lambda \geq \lambda_{0} .
\end{aligned}
$$

By (4.13) and (11.2), and noting that $u(0, x)=u(T, x) \equiv 0$, we get

$$
\begin{aligned}
& M(0, x)=2 \ell_{t}(0, x)\left[\theta(0, x) u_{t}(0, x)\right]^{2}=2 c T \lambda\left[\theta(0, x) u_{t}(0, x)\right]^{2}>0, \\
& M(T, x)=2 \ell_{t}(T, x)\left[\theta(T, x) u_{t}(T, x)\right]^{2}=-2 c T \lambda\left[\theta(T, x) u_{t}(T, x)\right]^{2}<0 .
\end{aligned}
$$

Combining (11.9) and (11.10), and noting the definition of $\Gamma_{+}$in (2.5), we obtain

$$
\begin{aligned}
\lambda \int_{Q}\left(\lambda^{2} v^{2}+v_{t}^{2}+\sum_{i, j} a^{i j} v_{i} v_{j}\right) d x d t \\
\leq C\left[\int_{Q} \theta^{2}|\mathcal{P} u|^{2} d x d t\right. \\
\left.\quad+\lambda \int_{0}^{T} \int_{\Gamma_{+}}\left(\sum_{i, j} a^{i j} \nu_{i} \nu_{j}\right)\left(\sum_{i^{\prime}, j^{\prime}} a^{i^{\prime} j^{\prime}} d_{i^{\prime}} \nu_{j^{\prime}}\right)\left|\frac{\partial v}{\partial \nu}\right|^{2} d x d t\right] .
\end{aligned}
$$

Recalling $u=\theta^{-1} v$ and $\theta=e^{\ell}$, noting (4.13) and (11.11), and noting (1.2) and $\left.u\right|_{\Sigma}=0$, we get

$$
\begin{aligned}
& \lambda \int_{Q} \theta^{2}\left(\lambda^{2} u^{2}+u_{t}^{2}+|\nabla u|^{2}\right) d x d t \\
& \leq C\left(\int_{Q} \theta^{2}|\mathcal{P} u|^{2} d x d t+\lambda \int_{0}^{T} \int_{\Gamma_{+}} \theta^{2}\left|\frac{\partial u}{\partial \nu}\right|^{2} d x d t\right) .
\end{aligned}
$$

Step 3. Let us estimate

$$
\int_{0}^{T} \int_{\Gamma_{+}} \theta^{2}\left|\frac{\partial u}{\partial \nu}\right|^{2} d x d t
$$

We choose a $g_{0} \in C^{1}\left(\bar{\Omega} ; \mathbb{R}^{n}\right)$ such that $g_{0}=\nu$ on $\Gamma$, and a $\rho \in C^{2}(\bar{\Omega} ;[0,1])$ such that (recall Condition 2.2 for $\delta$ )

$$
\begin{cases}\rho(x) \equiv 1, & x \in \mathcal{O}_{\delta / 3}\left(\Gamma_{+}\right) \cap \Omega, \\ \rho(x) \equiv 0, & x \in \Omega \backslash \mathcal{O}_{\delta / 2}\left(\Gamma_{+}\right) .\end{cases}
$$

Copyright (C) by SIAM. Unauthorized reproduction of this article is prohibited. 
Put

$$
g=g_{0} \rho \theta^{2} .
$$

Integrating (3.3) (in Lemma 3.2) in $Q$, with $g$ defined by (11.14) and $z$ replaced by $u$; using integration by parts; and noting (11.13), $u_{i}=\frac{\partial u}{\partial \nu} \nu_{i}$ on $\Sigma$ (which follows from $\left.\left.u\right|_{\Sigma}=0\right)$, and $u(0, x)=u(T, x) \equiv 0$, we get

$$
\begin{aligned}
& \int_{\Sigma}\left(\sum_{i, j} a^{i j} \nu_{i} \nu_{j}\right) \rho \theta^{2}\left|\frac{\partial u}{\partial \nu}\right|^{2} d x d t \\
& =\int_{Q} \sum_{j}\left[2(g \cdot \nabla u) \sum_{i} a^{i j} u_{i}+g^{j}\left(u_{t}^{2}-\sum_{i, k} a^{i k} u_{i} u_{k}\right)\right]_{j} d x d t \\
& =-\int_{Q}\left\{2\left[(\mathcal{P} u) g \cdot \nabla u-\left(u_{t} g \cdot \nabla u\right)_{t}+u_{t} g_{t} \cdot \nabla u-\sum_{i, j, k} a^{i j} u_{i} u_{k} \frac{\partial g^{k}}{\partial x_{j}}\right]\right. \\
& =-\int_{Q}\left\{2\left[(\mathcal{P} u) g \cdot \nabla u+u_{t} g \cdot \nabla u+u_{t} g_{t} \cdot \nabla u-\sum_{i, j, k}^{2} a^{i j} u_{i} u_{k} \frac{\partial g^{k}}{\partial x_{j}}\right]\right. \\
& \leq C\left[\frac{1}{\lambda}|\theta \mathcal{P} u|_{L^{2}(Q)}^{2}+\lambda \int_{0}^{T} \int_{\mathcal{O}_{\delta / 2}\left(\Gamma_{+}\right) \cap \Omega} \theta_{j}\left(u_{t}^{2}+|\nabla u|^{2}\right) d x d t\right]
\end{aligned}
$$

Step 4. Let us estimate

$$
\int_{0}^{T} \int_{\mathcal{O}_{\delta / 2}\left(\Gamma_{+}\right) \cap \Omega} \theta^{2}|\nabla u|^{2} d x d t
$$

Put

$$
\eta=\eta(t, x) \triangleq \rho_{1}^{2} \theta^{2}
$$

where $\rho_{1} \in C^{2}(\bar{\Omega} ;[0,1])$ satisfies

$$
\begin{cases}\rho_{1}(x) \equiv 1, & x \in \mathcal{O}_{\delta / 2}\left(\Gamma_{+}\right) \cap \Omega \\ \rho_{1}(x) \equiv 0, & x \in \Omega \backslash \omega\end{cases}
$$

By (1.3), we obtain

$$
\begin{aligned}
& \int_{Q} \eta u \mathcal{P} u d x d t=\int_{Q} \eta u\left(u_{t t}-\sum_{i, j}\left(a^{i j} u_{i}\right)_{j}\right) d x d t \\
& =-\int_{Q}\left[u_{t}\left(\eta_{t} u+\eta u_{t}\right)\right] d x d t+\int_{Q} \eta \sum_{i, j} a^{i j} u_{i} u_{j} d x d t+\int_{Q} u \sum_{i, j} a^{i j} u_{i} \eta_{j} d x d t
\end{aligned}
$$

Copyright $@$ by SIAM. Unauthorized reproduction of this article is prohibited. 
Hence, by (1.2) and (11.16)-(11.18), we find

$$
\begin{aligned}
& \int_{0}^{T} \int_{\mathcal{O}_{\delta / 2}\left(\Gamma_{+}\right) \cap \Omega} \theta^{2}|\nabla u|^{2} d x d t \\
& \leq C\left[\frac{1}{\lambda^{2}}|\theta \mathcal{P} u|_{L^{2}(Q)}^{2}+\int_{0}^{T} \int_{\omega} \theta^{2}\left(\lambda^{2} u^{2}+u_{t}^{2}\right) d x d t\right] .
\end{aligned}
$$

Finally, combining (11.12), (11.15), and (11.19), and noting (11.13), we get the desired estimate (5.3).

12. Appendix C. Proof of Proposition 6.1. We borrow some ideas from [18]. The proof is split into several steps.

Step 1. Let $\left\{\left\{\left(z_{m}^{i, j}, r_{1 m}^{i, j}, r_{2 m}^{i, j}, r_{m}^{i, j}\right)\right\}_{i=0}^{m}\right\}_{j=1}^{\infty} \subset \mathcal{A}_{a d}$ be a minimizing sequence of $J(\cdot)$. Because of the coercivity of the cost functional and noting that $z_{m}^{i, j}$ solves an elliptic equation, it can be shown that $\left\{\left\{\left(z_{m}^{i, j}, r_{1 m}^{i, j}, r_{2 m}^{i, j}, r_{m}^{i, j}\right)\right\}_{i=0}^{m}\right\}_{j=1}^{\infty}$ is bounded in $\mathcal{A}_{a d}$. Therefore, there exists a subsequence of $\left\{\left\{\left(z_{m}^{i, j}, r_{1 m}^{i, j}, r_{2 m}^{i, j}, r_{m}^{i, j}\right)\right\}_{i=0}^{m}\right\}_{j=1}^{\infty}$ converging weakly in $\left(H_{0}^{1}(\Omega) \times\left(L^{2}(\Omega)\right)^{3}\right)^{m+1}$ to some $\left\{\left(\hat{z}_{m}^{i}, \hat{r}_{1 m}^{i}, \hat{r}_{2 m}^{i}, \hat{r}_{m}^{i}\right)\right\}_{i=0}^{m} \in \mathcal{A}_{a d}$. Since the function $J$ is strictly convex, this element is the unique solution of (6.5). By (6.6) and the definition of $\mathcal{A}_{a d}$, it is obvious that $\hat{z}_{m}^{0}=\hat{z}_{m}^{m}=p_{m}^{0}=p_{m}^{m}=0$ in $\Omega$.

Step 2. Fix any $\delta_{0 m}^{i} \in H^{2}(\Omega) \cap H_{0}^{1}(\Omega), \delta_{1 m}^{i} \in L^{2}(\Omega)$, and $\delta_{2 m}^{i} \in L^{2}(\Omega)(i=$ $0,1,2, \ldots, m)$ with $\delta_{0 m}^{0}=\delta_{0 m}^{m}=\delta_{2 m}^{0}=\delta_{2 m}^{m} \equiv 0$ and $\delta_{1 m}^{0}=\delta_{1 m}^{1}$ in $\Omega$. For $\left(\lambda_{0}, \lambda_{1}, \lambda_{2}\right) \in \mathbb{R}^{3}$, put

$$
\left\{\begin{aligned}
r_{m}^{i} \triangleq & \frac{\hat{z}_{m}^{i+1}-2 \hat{z}_{m}^{i}+\hat{z}_{m}^{i-1}}{h^{2}}+\frac{\delta_{0 m}^{i+1}-2 \delta_{0 m}^{i}+\delta_{0 m}^{i-1}}{h^{2}} \lambda_{0} \\
& -\sum_{j_{1}, j_{2}=1}^{n} \partial_{x_{j_{2}}}\left(a^{j_{1} j_{2}} \partial_{x_{j_{1}}}\left(\hat{z}_{m}^{i}+\lambda_{0} \delta_{0 m}^{i}\right)\right) \\
& -\frac{\hat{r}_{1 m}^{i+1}-\hat{r}_{1 m}^{i}}{h}-\frac{\delta_{1 m}^{i+1}-\delta_{1 m}^{i}}{h} \lambda_{1}-\hat{r}_{2 m}^{i}-\lambda_{2} \delta_{2 m}^{i}-\lambda u_{m}^{i} e^{2 \lambda \phi_{m}^{i}}, \quad 1 \leq i \leq m-1 ; \\
r_{m}^{0}= & r_{m}^{m}=0 .
\end{aligned}\right.
$$

Then $\left\{\left(\hat{z}_{m}^{i}+\lambda_{0} \delta_{0 m}^{i}, \hat{r}_{1 m}^{i}+\lambda_{1} \delta_{1 m}^{i}, \hat{r}_{2 m}^{i}+\lambda_{2} \delta_{2 m}^{i}, r_{m}^{i}\right)\right\}_{i=0}^{m} \in \mathcal{A}_{a d}$. Define a function in $\mathbb{R}^{3}$ by

$$
g\left(\lambda_{0}, \lambda_{1}, \lambda_{2}\right)=J\left(\left\{\left(\hat{z}_{m}^{i}+\lambda_{0} \delta_{0 m}^{i}, \hat{r}_{1 m}^{i}+\lambda_{1} \delta_{1 m}^{i}, \hat{r}_{2 m}^{i}+\lambda_{2} \delta_{2 m}^{i}, r_{m}^{i}\right)\right\}_{i=0}^{m}\right) .
$$

Obviously $g$ has a minimum at $(0,0,0)$. Hence, $\nabla g(0,0,0)=0$. By $\frac{\partial g(0,0,0)}{\partial \lambda_{1}}=$ $\frac{\partial g(0,0,0)}{\partial \lambda_{2}}=0$, and noting that $\left\{\left(\hat{z}_{m}^{i}, \hat{r}_{1 m}^{i}, \hat{r}_{2 m}^{i}, \hat{r}_{m}^{i}\right)\right\}_{i=0}^{m}$ satisfy the first equation in (6.3), one gets

$$
\begin{aligned}
& -K \sum_{i=1}^{m-1} \int_{\Omega} \hat{r}_{m}^{i} \frac{\delta_{1 m}^{i+1}-\delta_{1 m}^{i}}{h} d x+\sum_{i=1}^{m} \int_{\Omega} \varrho \frac{\hat{r}_{1 m}^{i} \delta_{1 m}^{i}}{\lambda^{2}} e^{-2 \lambda \phi_{m}^{i}} d x=0 \\
& -K \sum_{i=1}^{m-1} \int_{\Omega} \hat{r}_{m}^{i} \delta_{2 m}^{i} d x+\sum_{i=1}^{m-1} \int_{\Omega} \varrho \frac{\hat{r}_{2 m}^{i} \delta_{2 m}^{i}}{\lambda^{4}} e^{-2 \lambda \phi_{m}^{i}} d x=0
\end{aligned}
$$

Copyright $\odot$ by SIAM. Unauthorized reproduction of this article is prohibited. 
which, combined with (6.6) and $p_{m}^{0}=p_{m}^{m}=\hat{r}_{2 m}^{m}=0$ in $\Omega$, gives (6.8). From $\frac{\partial g(0,0,0)}{\partial \lambda_{0}}=$ 0 , we obtain

$$
\begin{array}{r}
\sum_{i=1}^{m-1} \int_{\Omega}\left\{K \hat{r}_{m}^{i}\left[\frac{\delta_{0 m}^{i+1}-2 \delta_{0 m}^{i}+\delta_{0 m}^{i-1}}{h^{2}}-\sum_{j_{1}, j_{2}=1}^{n} \partial_{x_{j_{2}}}\left(a^{j_{1} j_{2}} \partial_{x_{j_{1}}} \delta_{0 m}^{i}\right)\right]\right. \\
\left.+\hat{z}_{m}^{i} \delta_{0 m}^{i} e^{-2 \lambda \phi_{m}^{i}}\right\} d x=0,
\end{array}
$$

which, combined with $p_{m}^{0}=p_{m}^{m}=\delta_{0 m}^{0}=\delta_{0 m}^{m}=0$ in $\Omega$, implies that $p_{m}^{i}=K \hat{r}_{m}^{i}$ is a weak solution of (6.9). By means of the regularity theory for elliptic equations of second order, one sees that $\hat{z}_{m}^{i}, p_{m}^{i} \in H^{2}(\Omega) \cap H_{0}^{1}(\Omega)$ for $1 \leq i \leq m-1$.

Step 3. Recalling that $\left\{\left(\hat{z}_{m}^{i}, \hat{r}_{1 m}^{i}, \hat{r}_{2 m}^{i}, \hat{r}_{m}^{i}\right)\right\}_{i=0}^{m}$ satisfy (6.3), and noting (6.7)-(6.9) and $p_{m}^{i}=K \hat{r}_{m}^{i}$, one gets

$$
\begin{aligned}
& 0=\sum_{i=1}^{m-1} \int_{\Omega}\left(\frac{\hat{z}_{m}^{i+1}-2 \hat{z}_{m}^{i}+\hat{z}_{m}^{i-1}}{h^{2}}-\sum_{j_{1}, j_{2}=1}^{n} \partial_{x_{j_{2}}}\left(a^{j_{1} j_{2}} \partial_{x_{j_{1}}} \hat{z}_{m}^{i}\right)\right. \\
& \left.-\frac{\hat{r}_{1 m}^{i+1}-\hat{r}_{1 m}^{i}}{h}-\hat{r}_{2 m}^{i}-\lambda u_{m}^{i} e^{2 \lambda \phi_{m}^{i}}-\hat{r}_{m}^{i}\right) p_{m}^{i} d x \\
& =\sum_{i=1}^{m-1} \int_{\Omega}\left(\frac{p_{m}^{i+1}-2 p_{m}^{i}+p_{m}^{i-1}}{h^{2}}-\sum_{j_{1}, j_{2}=1}^{n} \partial_{x_{j_{2}}}\left(a^{j_{1} j_{2}} \partial_{x_{j_{1}}} p_{m}^{i}\right)\right) \hat{z}_{m}^{i} d x \\
& +\sum_{i=1}^{m} \int_{\Omega} \frac{p_{m}^{i}-p_{m}^{i-1}}{h} \hat{r}_{1 m}^{i} d x-\sum_{i=1}^{m-1} \int_{\Omega}\left(\hat{r}_{2 m}^{i}+\lambda u_{m}^{i} e^{2 \lambda \phi_{m}^{i}}+\hat{r}_{m}^{i}\right) p_{m}^{i} d x \\
& =-\sum_{i=1}^{m-1}\left[\int_{\Omega}\left|\hat{z}_{m}^{i}\right|^{2} e^{-2 \lambda \phi_{m}^{i}} d x+\int_{\Omega} \varrho\left(\frac{\left|\hat{r}_{1 m}^{i}\right|^{2}}{\lambda^{2}}+\frac{\left|\hat{r}_{2 m}^{i}\right|^{2}}{\lambda^{4}}\right) e^{-2 \lambda \phi_{m}^{i}} d x\right. \\
& \left.+K \int_{\Omega}\left|\hat{r}_{m}^{i}\right|^{2} d x\right]-\int_{\Omega} \varrho \frac{\left|\hat{r}_{1 m}^{m}\right|^{2}}{\lambda^{2}} e^{-2 \lambda \phi_{m}^{m}} d x-\lambda \sum_{i=1}^{m-1} \int_{\Omega} u_{m}^{i} e^{2 \lambda \phi_{m}^{i}} p_{m}^{i} d x .
\end{aligned}
$$

Using the Hölder inequality, by (12.2) and (6.8) we conclude that there is a constant $C=C(K, \lambda)>0$, independent of $m$, such that

$$
\begin{aligned}
& \sum_{i=1}^{m-1}\left[\int_{\Omega}\left|\hat{z}_{m}^{i}\right|^{2} e^{-2 \lambda \phi_{m}^{i}} d x+\int_{\Omega} \varrho\left(\frac{\left|\hat{r}_{1 m}^{i}\right|^{2}}{\lambda^{2}}+\frac{\left|\hat{r}_{2 m}^{i}\right|^{2}}{\lambda^{4}}\right) e^{-2 \lambda \phi_{m}^{i}} d x+K \int_{\Omega}\left|\hat{r}_{m}^{i}\right|^{2} d x\right] \\
& \quad+\int_{\Omega} \varrho \frac{\left|\hat{r}_{1 m}^{m}\right|^{2}}{\lambda^{2}} e^{-2 \lambda \phi_{m}^{m}} d x \\
& \leq C \sum_{i=1}^{m-1} \int_{\Omega}\left|u_{m}^{i}\right|^{2} e^{2 \lambda \phi_{m}^{i}} d x .
\end{aligned}
$$

This yields (6.10).

Step 4. Noting that (6.9) holds for $i=1,2, \ldots, m-1$, and that $p_{m}^{0}=\hat{z}_{m}^{0}=p_{m}^{m}=$ $\hat{z}_{m}^{m}=0$, one gets

Copyright (c) by SIAM. Unauthorized reproduction of this article is prohibited. 


$$
\begin{array}{ll}
\frac{p_{m}^{3}-4 p_{m}^{2}+5 p_{m}^{1}}{h^{4}}-\sum_{j_{1}, j_{2}=1}^{n} \partial_{x_{j_{2}}}\left(a^{j_{1} j_{2}} \partial_{x_{j_{1}}} \frac{\left(p_{m}^{2}-2 p_{m}^{1}-p_{m}^{0}\right)}{h^{2}}\right) & \\
+\frac{\hat{z}_{m}^{2} e^{-2 \lambda \phi_{m}^{2}}-2 \hat{z}_{m}^{1} e^{-2 \lambda \phi_{m}^{1}}+\hat{z}_{m}^{0} e^{-2 \lambda \phi_{m}^{0}}}{h^{2}}=0 & \text { in } \Omega, \\
\frac{5 p_{m}^{m-1}-4 p_{m}^{m-2}+p_{m}^{m-3}}{h^{4}}-\sum_{j_{1}, j_{2}=1}^{n} \partial_{x_{j_{2}}}\left(a^{j_{1} j_{2}} \partial_{x_{j_{1}}} \frac{\left(p_{m}^{m}-2 p_{m}^{m-1}+p_{m}^{m-2}\right)}{h^{2}}\right) & \text { in } \Omega, \\
+\frac{\hat{z}_{m}^{m} e^{-2 \lambda \phi_{m}^{m}}-2 \hat{z}_{m}^{m-1} e^{-2 \lambda \phi_{m}^{m-1}}+\hat{z}_{m}^{m-2} e^{-2 \lambda \phi_{m}^{m-2}}}{h^{2}}=0 &
\end{array}
$$

and for $i=2, \ldots, m-2$,

$$
\begin{array}{ll}
\frac{p_{m}^{i+2}-4 p_{m}^{i+1}+6 p_{m}^{i}-4 p_{m}^{i-1}+p_{m}^{i-2}}{h^{4}} & \\
-\sum_{j_{1}, j_{2}=1}^{n} \partial_{x_{j_{2}}}\left(a^{j_{1} j_{2}} \partial_{x_{j_{1}}} \frac{\left(p_{m}^{i+1}-2 p_{m}^{i}+p_{m}^{i-1}\right)}{h^{2}}\right) & \\
+\frac{\hat{z}_{m}^{i+1} e^{-2 \lambda \phi_{m}^{i+1}}-2 \hat{z}_{m}^{i} e^{-2 \lambda \phi_{m}^{i}}+\hat{z}_{m}^{i-1} e^{-2 \lambda \phi_{m}^{i-1}}}{h^{2}}=0 & \text { in } \Omega .
\end{array}
$$

By (6.3), we find

$$
\begin{aligned}
0=\sum_{i=1}^{m-1} & \int_{\Omega}\left(\frac{\hat{z}_{m}^{i+1}-2 \hat{z}_{m}^{i}+\hat{z}_{m}^{i-1}}{h^{2}}-\sum_{j_{1}, j_{2}=1}^{n} \partial_{x_{j_{2}}}\left(a^{j_{1} j_{2}} \partial_{x_{j_{1}}} \hat{z}_{m}^{i}\right)\right. \\
& \left.\quad-\frac{\hat{r}_{1 m}^{i+1}-\hat{r}_{1 m}^{i}}{h}-\hat{r}_{2 m}^{i}-\lambda u_{m}^{i} e^{2 \lambda \phi_{m}^{i}}-\hat{r}_{m}^{i}\right) \frac{\left(p_{m}^{i+1}-2 p_{m}^{i}+p_{m}^{i-1}\right)}{h^{2}} d x .
\end{aligned}
$$

Noting $\hat{z}_{m}^{0}=\hat{z}_{m}^{m}=p_{m}^{0}=p_{m}^{m}=0$ again, and using (12.3)-(12.4), we arrive at

$$
\begin{aligned}
& \sum_{i=1}^{m-1} \int_{\Omega} \frac{\left(\hat{z}_{m}^{i+1}-2 \hat{z}_{m}^{i}+\hat{z}_{m}^{i-1}\right)}{h^{2}} \frac{\left(p_{m}^{i+1}-2 p_{m}^{i}+p_{m}^{i-1}\right)}{h^{2}} d x \\
& =\sum_{i=2}^{m-1} \int_{\Omega} \hat{z}_{m}^{i} \frac{\left(p_{m}^{i}-2 p_{m}^{i-1}+p_{m}^{i-2}\right)}{h^{4}} d x-2 \sum_{i=1}^{m-1} \int_{\Omega} \hat{z}_{m}^{i} \frac{\left(p_{m}^{i+1}-2 p_{m}^{i}+p_{m}^{i-1}\right)}{h^{4}} d x \\
& \quad+\sum_{i=1}^{m-2} \int_{\Omega} \hat{z}_{m}^{i} \frac{\left(p_{m}^{i+2}-2 p_{m}^{i+1}+p_{m}^{i}\right)}{h^{4}} d x \\
& =\int_{\Omega} \hat{z}_{m}^{1} \frac{\left(p_{m}^{3}-4 p_{m}^{2}+5 p_{m}^{1}\right)}{h^{4}} d x+\int_{\Omega} \hat{z}_{m}^{m-1} \frac{\left(5 p_{m}^{m-1}-4 p_{m}^{m-2}+p_{m}^{m-3}\right)}{h^{4}} d x \\
& \quad+\sum_{i=2}^{m-2} \int_{\Omega} \hat{z}_{m}^{i} \frac{\left(p_{m}^{i+2}-4 p_{m}^{i+1}+6 p_{m}^{i}-4 p_{m}^{i-1}+p_{m}^{i-2}\right)}{h^{4}} d x \\
& =\sum_{i=1}^{m-1} \int_{\Omega} \hat{z}_{m}^{i}\left\{\sum_{j_{1}, j_{2}=1}^{n} \partial_{x_{j_{2}}}\left[a^{j_{1} j_{2}} \partial_{x_{j_{1}}} \frac{\left(p_{m}^{i+1}-2 p_{m}^{i}+p_{m}^{i-1}\right)}{h^{2}}\right]\right. \\
& \left.-\frac{\hat{z}_{m}^{i+1} e^{-2 \lambda \phi_{m}^{i+1}}-2 \hat{z}_{m}^{i} e^{-2 \lambda \phi_{m}^{i}}+\hat{z}_{m}^{i-1} e^{-2 \lambda \phi_{m}^{i-1}}}{h^{2}}\right\} d x .
\end{aligned}
$$

Copyright $@$ by SIAM. Unauthorized reproduction of this article is prohibited. 
Next, noting $\left.z_{m}^{i}\right|_{\Gamma}=\left.p_{m}^{i}\right|_{\Gamma}=0$, for $0 \leq i \leq m$, one has

$$
\begin{aligned}
& \sum_{i=1}^{m-1} \int_{\Omega}\left(\sum_{j_{1}, j_{2}=1}^{n} \partial_{x_{j_{2}}}\left(a^{j_{1} j_{2}} \partial_{x_{j_{1}}} \hat{z}_{m}^{i}\right)\right) \frac{\left(p_{m}^{i+1}-2 p_{m}^{i}+p_{m}^{i-1}\right)}{h^{2}} d x \\
& =\sum_{i=1}^{m-1} \int_{\Omega} \hat{z}_{m}^{i} \sum_{j_{1}, j_{2}=1}^{n} \partial_{x_{j_{2}}}\left(a^{j_{1} j_{2}} \partial_{x_{j_{1}}} \frac{\left(p_{m}^{i+1}-2 p_{m}^{i}+p_{m}^{i-1}\right)}{h^{2}}\right) d x .
\end{aligned}
$$

Combining (12.5)-(12.7), we obtain

$$
\begin{aligned}
0= & -\sum_{i=1}^{m-1} \int_{\Omega}\left[\hat{z}_{m}^{i} \frac{\left(\hat{z}_{m}^{i+1} e^{-2 \lambda \phi_{m}^{i+1}}-2 \hat{z}_{m}^{i} e^{-2 \lambda \phi_{m}^{i}}+\hat{z}_{m}^{i-1} e^{-2 \lambda \phi_{m}^{i-1}}\right)}{h^{2}}\right. \\
& \left.+\left(\frac{\hat{r}_{1 m}^{i+1}-\hat{r}_{1 m}^{i}}{h}+\hat{r}_{2 m}^{i}+\lambda u_{m}^{i} e^{2 \lambda \phi_{m}^{i}}+\hat{r}_{m}^{i}\right) \frac{\left(p_{m}^{i+1}-2 p_{m}^{i}+p_{m}^{i-1}\right)}{h^{2}}\right] d x .
\end{aligned}
$$

By Proposition 3.5 and noting $p_{m}^{i}=K \hat{r}_{m}^{i}$, one has

$$
\begin{gathered}
-\sum_{i=1}^{m-1} \int_{\Omega}\left[\hat{z}_{m}^{i} \frac{\left(\hat{z}_{m}^{i+1} e^{-2 \lambda \phi_{m}^{i+1}}-2 \hat{z}_{m}^{i} e^{-2 \lambda \phi_{m}^{i}}+\hat{z}_{m}^{i-1} e^{-2 \lambda \phi_{m}^{i-1}}\right)}{h^{2}}+\hat{r}_{m}^{i} \frac{\left(p_{m}^{i+1}-2 p_{m}^{i}+p_{m}^{i-1}\right)}{h^{2}}\right] d x \\
=\sum_{i=0}^{m-1} \int_{\Omega}\left[\frac{\left(\hat{z}_{m}^{i+1}-\hat{z}_{m}^{i}\right)}{h} \frac{\left(\hat{z}_{m}^{i+1} e^{-2 \lambda \phi_{m}^{i+1}}-\hat{z}_{m}^{i} e^{-2 \lambda \phi_{m}^{i}}\right)}{h}+K \frac{\left(\hat{r}_{m}^{i+1}-\hat{r}_{m}^{i}\right)^{2}}{h^{2}}\right] d x \\
=\sum_{i=0}^{m-1} \int_{\Omega}\left[\frac{\left(\hat{z}_{m}^{i+1}-\hat{z}_{m}^{i}\right)^{2}}{h^{2}} e^{-2 \lambda \phi_{m}^{i}}+\frac{\left(\hat{z}_{m}^{i+1}-\hat{z}_{m}^{i}\right)}{h} \frac{\left(e^{-2 \lambda \phi_{m}^{i+1}}-e^{-2 \lambda \phi_{m}^{i}}\right)}{h} \hat{z}_{m}^{i+1}\right. \\
\left.+K \frac{\left(\hat{r}_{m}^{i+1}-\hat{r}_{m}^{i}\right)^{2}}{h^{2}}\right] d x .
\end{gathered}
$$

Further, by (6.8), and using Proposition 3.5 again, we find

$$
\begin{aligned}
& -\sum_{i=1}^{m-1} \int_{\Omega}\left(\frac{\hat{r}_{1 m}^{i+1}-\hat{r}_{1 m}^{i}}{h}+\hat{r}_{2 m}^{i}+\lambda u_{m}^{i} e^{2 \lambda \phi_{m}^{i}}\right) \frac{\left(p_{m}^{i+1}-2 p_{m}^{i}+p_{m}^{i-1}\right)}{h^{2}} d x \\
& =-\sum_{i=1}^{m-1} \int_{\Omega}\left(\frac{\hat{r}_{1 m}^{i+1}-\hat{r}_{1 m}^{i}}{h}+\lambda u_{m}^{i} e^{2 \lambda \phi_{m}^{i}}\right) \frac{1}{h}\left(\frac{p_{m}^{i+1}-p_{m}^{i}}{h}-\frac{p_{m}^{i}-p_{m}^{i-1}}{h}\right) d x \\
& +\sum_{i=0}^{m-1} \int_{\Omega} \frac{\left(\hat{r}_{2 m}^{i+1}-\hat{r}_{2 m}^{i}\right)}{h} \frac{\left(p_{m}^{i+1}-p_{m}^{i}\right)}{h} d x \\
& =\sum_{i=1}^{m-1} \int_{\Omega} \frac{\varrho}{\lambda^{2}}\left(\frac{\hat{r}_{1 m}^{i+1}-\hat{r}_{1 m}^{i}}{h}+\lambda u_{m}^{i} e^{2 \lambda \phi_{m}^{i}}\right) \frac{\left(\hat{r}_{1 m}^{i+1} e^{-2 \lambda \phi_{m}^{i+1}}-\hat{r}_{1 m}^{i} e^{-2 \lambda \phi_{m}^{i}}\right)}{h} d x \\
& +\sum_{i=0}^{m-1} \int_{\Omega} \frac{\varrho}{\lambda^{4}} \frac{\left(\hat{r}_{2 m}^{i+1}-\hat{r}_{2 m}^{i}\right)}{h} \frac{\left(\hat{r}_{2 m}^{i+1} e^{-2 \lambda \phi_{m}^{i+1}}-\hat{r}_{2 m}^{i} e^{-2 \lambda \phi_{m}^{i}}\right)}{h} d x \\
& =\sum_{i=1}^{m-1} \int_{\Omega} \frac{\varrho}{\lambda^{2}}\left[\frac{\left(\hat{r}_{1 m}^{i+1}-\hat{r}_{1 m}^{i}\right)^{2}}{h^{2}} e^{-2 \lambda \phi_{m}^{i}}\right. \\
& \left.+\frac{\left(\hat{r}_{1 m}^{i+1}-\hat{r}_{1 m}^{i}\right)}{h} \frac{\left(e^{-2 \lambda \phi_{m}^{i+1}}-e^{-2 \lambda \phi_{m}^{i}}\right)}{h} \hat{r}_{1 m}^{i+1}\right] d x
\end{aligned}
$$

Copyright $($ C by SIAM. Unauthorized reproduction of this article is prohibited. 


$$
\begin{gathered}
+\lambda \sum_{i=1}^{m-1} \int_{\Omega} \frac{\varrho}{\lambda^{2}} u_{m}^{i}\left[\frac{\left(\hat{r}_{1 m}^{i+1}-\hat{r}_{1 m}^{i}\right)}{h} e^{-2 \lambda \phi_{m}^{i}}+\frac{\left(e^{-2 \lambda \phi_{m}^{i+1}}-e^{-2 \lambda \phi_{m}^{i}}\right)}{h} \hat{r}_{1 m}^{i+1}\right] d x \\
+\sum_{i=0}^{m-1} \int_{\Omega} \frac{\varrho}{\lambda^{4}}\left[\frac{\left(\hat{r}_{2 m}^{i+1}-\hat{r}_{2 m}^{i}\right)^{2}}{h^{2}} e^{-2 \lambda \phi_{m}^{i}}\right. \\
\left.+\frac{\left(\hat{r}_{2 m}^{i+1}-\hat{r}_{2 m}^{i}\right)}{h} \frac{\left(e^{-2 \lambda \phi_{m}^{i+1}}-e^{-2 \lambda \phi_{m}^{i}}\right)}{h} \hat{r}_{2 m}^{i+1}\right] d x .
\end{gathered}
$$

Combining (12.8)-(12.10), and noting that $\hat{r}_{1 m}^{1}=\hat{r}_{1 m}^{0}, u_{m}^{0}=0$, we end up with

$$
\begin{aligned}
\sum_{i=0}^{m-1} \int_{\Omega}[ & \frac{\left(\hat{z}_{m}^{i+1}-\hat{z}_{m}^{i}\right)^{2}}{h^{2}} e^{-2 \lambda \phi_{m}^{i}}+\frac{\varrho}{\lambda^{2}} \frac{\left(\hat{r}_{1 m}^{i+1}-\hat{r}_{1 m}^{i}\right)^{2}}{h^{2}} e^{-2 \lambda \phi_{m}^{i}} \\
& \left.+\frac{\varrho}{\lambda^{4}} \frac{\left(\hat{r}_{2 m}^{i+1}-\hat{r}_{2 m}^{i}\right)^{2}}{h^{2}} e^{-2 \lambda \phi_{m}^{i}}+K \frac{\left(\hat{r}_{m}^{i+1}-\hat{r}_{m}^{i}\right)^{2}}{h^{2}}\right] d x \\
= & -\sum_{i=0}^{m-1} \int_{\Omega} \frac{\left(\hat{z}_{m}^{i+1}-\hat{z}_{m}^{i}\right)}{h} \frac{\left(e^{-2 \lambda \phi_{m}^{i+1}}-e^{-2 \lambda \phi_{m}^{i}}\right)}{h} \hat{z}_{m}^{i+1} d x \\
& -\sum_{i=1}^{m-1} \int_{\Omega} \frac{\varrho}{\lambda^{2}} \frac{\left(\hat{r}_{1 m}^{i+1}-\hat{r}_{1 m}^{i}\right)}{h} \frac{\left(e^{-2 \lambda \phi_{m}^{i+1}}-e^{-2 \lambda \phi_{m}^{i}}\right)}{h} \hat{r}_{1 m}^{i+1} d x \\
& -\lambda \sum_{i=1}^{m-1} \int_{\Omega} \frac{\varrho}{\lambda^{2}} u_{m}^{i}\left[\frac{\left(\hat{r}_{1 m}^{i+1}-\hat{r}_{1 m}^{i}\right)}{h} e^{-2 \lambda \phi_{m}^{i}}+\frac{\left(e^{-2 \lambda \phi_{m}^{i+1}}-e^{\left.-2 \lambda \phi_{m}^{i}\right)}\right.}{h} \hat{r}_{1 m}^{i+1}\right] d x \\
& -\sum_{i=0}^{m-1} \int_{\Omega} \frac{\varrho}{\lambda^{4}} \frac{\left(\hat{r}_{2 m}^{i+1}-\hat{r}_{2 m}^{i}\right)}{h} \frac{\left(e^{-2 \lambda \phi_{m}^{i+1}}-e^{-2 \lambda \phi_{m}^{i}}\right)}{h} \hat{r}_{2 m}^{i+1} d x .
\end{aligned}
$$

Using the Hölder inequality and noting that $\phi$ is a smooth function, from (12.11) we conclude that there is a positive constant $C=C(K, \lambda)$, independent of $m$, such that

$$
\begin{aligned}
& \sum_{i=0}^{m-1} \int_{\Omega} {\left[\frac{\left(\hat{z}_{m}^{i+1}-\hat{z}_{m}^{i}\right)^{2}}{h^{2}} e^{-2 \lambda \phi_{m}^{i}}+\frac{\varrho}{\lambda^{2}} \frac{\left(\hat{r}_{1 m}^{i+1}-\hat{r}_{1 m}^{i}\right)^{2}}{h^{2}} e^{-2 \lambda \phi_{m}^{i}}\right.} \\
&\left.+\frac{\varrho}{\lambda^{4}} \frac{\left(\hat{r}_{2 m}^{i+1}-\hat{r}_{2 m}^{i}\right)^{2}}{h^{2}} e^{-2 \lambda \phi_{m}^{i}}+K \frac{\left(\hat{r}_{m}^{i+1}-\hat{r}_{m}^{i}\right)^{2}}{h^{2}}\right] d x \\
& \leq C\left[\sum_{i=1}^{m-1} \int_{\Omega}\left(\left|\hat{z}_{m}^{i}\right|^{2}+\left|\hat{r}_{1 m}^{i}\right|^{2}+\left|\hat{r}_{2 m}^{i}\right|^{2}+K\left|\hat{r}_{m}^{i}\right|^{2}+\left|u_{m}^{i}\right|^{2}\right) d x\right. \\
&\left.+\int_{\Omega}\left|\hat{r}_{1 m}^{m}\right|^{2} d x\right] .
\end{aligned}
$$

Finally, combining (12.12) and (6.10), and recalling that $u \in C\left([0, T] ; L^{2}(\Omega)\right)$, we establish the desired estimate (6.11). This completes the proof of Proposition 6.1 .

Acknowledgments. The authors acknowledge the anonymous referees for their comments which led to this improved version. The third author also thanks Professor M. Yamamoto for stimulating discussion.

Copyright $@$ by SIAM. Unauthorized reproduction of this article is prohibited. 


\section{REFERENCES}

[1] R. A. Adams, Sobolev Spaces, Academic Press, New York, 1975.

[2] C. Bardos, G. Lebeau, And J. Rauch, Sharp sufficient conditions for the observation, control, and stabilization of waves from the boundary, SIAM J. Control Optim., 30 (1992), pp. 1024-1065.

[3] P. Cannarsa, V. Komornik, and P. Loreti, One-sided and internal controllability of semilinear wave equations with infinitely iterated logarithms, Discrete Contin. Dyn. Syst. B, 8 (2002), pp. 745-756.

[4] T. Cazenave and A. Haraux, Equations d'évolution avec non-linéarité logarithmique, Ann. Fac. Sci. Toulouse, 2 (1980), pp. 21-51.

[5] W. C. Chewning, Controllability of the nonlinear wave equation in several space variables, SIAM J. Control Optim., 14 (1976), pp. 19-25.

[6] M. CIRINÀ, Boundary controllability of nonlinear hyperbolic systems, SIAM J. Control, 7 (1969), pp. 198-212.

[7] A. Doubova and A. Osses, Rotated weights in global Carleman estimates applied to an inverse problem for the wave equation, Inverse Problems, 33 (2006), pp. 265-296.

[8] T. Duyckaerts, X. Zhang, and E. Zuazua, On the optimality of the observability inequalities for parabolic and hyperbolic systems with potentials, Ann. Inst. H. Poincaré Anal. Non Linéaire, to appear.

[9] O. Yu. Émanullov, Boundary controllability of semilinear evolution equations, Russian Math. Surveys, 44 (1989), pp. 183-184.

[10] H. O. FatTorini, Local controllability of a nonlinear wave equation, Math. Systems Theory, 9 (1975), pp. 30-45.

[11] E. Fernández-Cara And E. Zuazua, The cost of approximate controllability for heat equations: The linear case, Adv. Differential Equations, 5 (2000), pp. 465-514.

[12] X. FU, A weighted identity for partial differential operators of second order and its applications, C. R. Math. Acad. Sci. Paris, 342 (2006), pp. 579-584.

[13] X. Fu, J. Yong, And X. Zhang, Exact Controllability of the Heat Equation with Hyperbolic Memory Kernel in Anisotropic and Non-Homogeneous Media, preprint, 2005.

[14] A. V. Fursikov and O. Yu. Imanuvilov, Controllability of Evolution Equations, Lecture Notes Series 34, Research Institute of Mathematics, Seoul National University, Seoul, Korea, 1994.

[15] L. Hörmander, On the Nash-Moser implicit function theorem, Ann. Acad. Sci. Fenn. Ser. A I Math., 10 (1985), pp. 255-259.

[16] L. Hörmander, Linear Partial Differential Operators, Springer-Verlag, Berlin, 1969.

[17] L. Hörmander, The Analysis of Linear Partial Differential Operators IV, Springer, Berlin, 1985.

[18] O. Yu. Imanuvilov, On Carleman estimates for hyperbolic equations, Asymptot. Anal., 32 (2002), pp. 185-220.

[19] V. Isakov and M. Yamamoto, Carleman estimate with the Neumann boundary condition and its applications to the observability inequality and inverse problems, in Differential Geometric Methods in the Control of Partial Differential Equations (Boulder, CO, 1999), Contemp. Math. 268, AMS, Providence, RI, 2000, pp. 191-225.

[20] M. Kazemi And M. V. Klibanov, Stability estimates for ill-posed Cauchy problem involving hyperbolic equations and inequalities, Appl. Anal., 50 (1993), pp. 93-102.

[21] M. V. Klibanov and J. Malinsky, Newton-Kantorovich method for 3-dimensional potential inverse scattering problem and stability of the hyperbolic Cauchy problem with time dependent data, Inverse Problems, 7 (1991), pp. 577-595.

[22] M. V. Klibanov and A. Timonov, Carleman Estimates for Coefficient Inverse Problems and Numerical Applications, VSP, Utrecht, 2004.

[23] I. LASIECKA AND R. TRIGGiani, Exact controllability of semilinear abstract systems with application to waves and plates boundary control problems, Appl. Math. Optim., 23 (1991), pp. 109-154.

[24] I. Lasiecka, R. Triggiani, And X. Zhang, Nonconservative wave equations with unobserved Neumann B.C.: Global uniqueness and observability in one shot, in Differential Geometric Methods in the Control of Partial Differential Equations (Boulder, CO, 1999), Contemp. Math. 268, AMS, Providence, RI, 2000, pp. 227-325.

[25] M. M. Lavrent'ev, V. G. Romanov, and S. P. Shishatskit, Ill-posed problems of mathematical physics and analysis, translated from the Russian by J. R. Schulenberger, Transl. Math. Monogr. 64, AMS, Providence, RI, 1986.

Copyright $@$ by SIAM. Unauthorized reproduction of this article is prohibited. 
[26] L. Li And X. Zhang, Exact controllability for semilinear wave equations, J. Math. Anal. Appl., 250 (2000), pp. 589-597.

[27] T.-T. Li AND B.-P. RAO, Exact boundary controllability for quasi-linear hyperbolic systems, SIAM J. Control Optim., 41 (2003), pp. 1748-1755.

[28] X. Li and J. Yong, Optimal Control Theory for Infinite-Dimensional Systems, Systems Control Found. Appl., Birkhäuser Boston, Inc., Boston, MA, 1995.

[29] J.-L. Lions, Contrôlabilité exacte, perturbations et stabilisation de systèmes distribués, Tome 1. Contrôlabilité exacte, Rech. Math. Appl. 8, Masson, Paris, 1988.

[30] P. Martinez and J. Vancostenoble, Exact controllability in "arbitrarily short time" of the semilinear wave equation, Discrete Contin. Dyn. Syst. B, 9 (2003), pp. 901-924.

[31] A. PAZY, Semigroups of Linear Operators and Applications to Partial Differential Equations, Appl. Math. Sci. 44, Springer-Verlag, New York, 1983.

[32] J. Rauch, X. Zhang, and E. Zuazua, Polynomial decay of a hyperbolic-parabolic coupled system, J. Math. Pures Appl., 84 (2005), pp. 407-470.

[33] D. L. Russell, Controllability and stabilizability theory for linear partial differential equations: Recent progress and open questions, SIAM Rev., 20 (1978), pp. 639-739.

[34] T. I. SEIDMAN, Invariance of the reachable set under nonlinear perturbations, SIAM J. Control Optim., 25 (1987), pp. 1173-1191.

[35] D. TATARU, Carleman estimates and unique continuation for solutions to boundary value problems, J. Math. Pures Appl., 75 (1996), pp. 367-408.

[36] X. Zhang, Explicit observability estimate for the wave equation with potential and its application, R. Soc. Lond. Proc. Ser. A Math. Phys. Eng. Sci., 456 (2000), pp. 1101-1115.

[37] X. ZhANG, Explicit observability inequalities for the wave equation with lower order terms by means of Carleman inequalities, SIAM J. Control Optim., 39 (2000), pp. 812-834.

[38] X. Zhang, Exact controllability of the semilinear plate equations, Asymptot. Anal., 27 (2001), pp. $95-125$.

[39] X. Zhang, Exact Controllability of Semi-Linear Distributed Parameter Systems, Higher Education Press, Beijing, 2004 (in Chinese).

[40] X. Zhang And E. Zuazua, Decay of solutions of the system of thermoelasticity of type III, Commun. Contemp. Math., 5 (2003), pp. 25-83.

[41] X. Zhang And E. Zunzua, Polynomial decay and control of a 1-d hyperbolic-parabolic coupled system, J. Differential Equations, 204 (2004), pp. 380-438.

[42] X. Zhang And E. Zuazua, Exact controllability of the semi-linear wave equation, in Sixty Open Problems in the Mathematics of Systems and Control, V. D. Blondel and A. Megretski, eds., Princeton University Press, Princeton, NJ, 2004, pp. 173-178.

[43] E. ZuazuA, Exact controllability for the semilinear wave equation, J. Math. Pures Appl., 69 (1990), pp. 1-31.

[44] E. ZuAzuA, Exact boundary controllability for the semilinear wave equation, in Nonlinear Partial Differential Equations and their Applications, Collège de France Seminar, Vol. X (Paris, 1987-1988), Pitman Res. Notes Math. Ser. 220, Longman Sci. Tech., Harlow, 1991, pp. 357-391.

[45] E. ZuAZUA, Exact controllability for semilinear wave equations in one space dimension, Ann. Inst. H. Poincaré Anal. Non Linéaire, 10 (1993), pp. 109-129.

Copyright (c) by SIAM. Unauthorized reproduction of this article is prohibited. 